\title{
Relative oscillation theory for matrix Sturm-Liouville difference equations extended
}

Julia V Elyseeva*

\section{"Correspondence:} elyseeva@mtu-net.ru Department of Applied Mathematics, Moscow State University of Technology, Vadkovskii per. 3a, Moscow, 101472, Russia

\begin{abstract}
In this article we establish relative oscillation theorems for two discrete matrix Sturm-Liouville eigenvalue problems with Dirichlet boundary conditions and nonlinear dependence on the spectral parameter $\lambda$. This nonlinear dependence on $\lambda$ is allowed both in the leading coefficients and in the potentials. Relative oscillation theory rather than measuring the spectrum of one single problem measures the difference between the spectra of two different problems. This is done by replacing focal points of conjoined bases of one problem by matrix analogs of weighted zeros of Wronskians of conjoined bases of two different problems.
\end{abstract}

MSC: 39A21;39A12

Keywords: discrete eigenvalue problem; matrix Sturm-Liouville equations; relative oscillation theory

\section{Introduction}

We consider the discrete matrix Sturm-Liouville spectral problems

$$
\begin{aligned}
& \Delta\left(R_{i}(\lambda) \Delta x_{i}(\lambda)\right)-Q_{i}(\lambda) x_{i+1}(\lambda)=0, \quad i \in[0, N-1], \\
& x_{0}(\lambda)=x_{N+1}(\lambda)=0, \quad \operatorname{det} R_{i}(\lambda) \neq 0, \quad i \in[0, N]
\end{aligned}
$$

and

$$
\begin{aligned}
& \Delta\left(\hat{R}_{i}(\lambda) \Delta \hat{x}_{i}(\lambda)\right)-\hat{Q}_{i}(\lambda) \hat{x}_{i+1}(\lambda)=0, \quad i \in[0, N-1], \\
& \hat{x}_{0}(\lambda)=\hat{x}_{N+1}(\lambda)=0, \quad \operatorname{det} \hat{R}_{i}(\lambda) \neq 0, \quad i \in[0, N],
\end{aligned}
$$

where $\Delta x_{i}=x_{i+1}-x_{i}, x_{i}(\lambda) \in \mathbb{R}^{n}, n \geq 1, \lambda \in \mathbb{R}$ is the spectral parameter, and the real symmetric $n \times n$ matrix-valued functions $R_{i}(\lambda), \hat{R}_{i}(\lambda), Q_{i}(\lambda), \hat{Q}_{i}(\lambda), i \in[0, N]$ are differentiable in the variable $\lambda$ and obey the conditions

$$
\begin{array}{rlrl}
\frac{d}{d \lambda} R_{i}(\lambda) & \leq 0, & \frac{d}{d \lambda} Q_{i}(\lambda) \leq 0, & i \in[0, N], \\
\frac{d}{d \lambda} \hat{R}_{i}(\lambda) \leq 0, & \frac{d}{d \lambda} \hat{Q}_{i}(\lambda) \leq 0, & i \in[0, N] .
\end{array}
$$

@2013 Elyseeva; licensee Springer. This is an Open Access article distributed under the terms of the Creative Commons Attribution License (http://creativecommons.org/licenses/by/2.0), which permits unrestricted use, distribution, and reproduction in any medium, provided the original work is properly cited. 
Conditions $(1.3)$ imply $[1,2]$ that problem $(1.1)$ is a special case of the discrete symplectic eigenvalue problem

$$
\begin{aligned}
& y_{i+1}(\lambda)=W_{i}(\lambda) y_{i}(\lambda), \quad y_{i}(\lambda)=\left[x_{i}^{T}, u_{i}^{T}\right]^{T} \in \mathbb{R}^{2 n}, \quad i=0, \ldots, N, \\
& x_{0}(\lambda)=x_{N+1}(\lambda)=0, \\
& \Psi_{i}(\lambda)=J \frac{d}{d \lambda}\left(W_{i}(\lambda)\right) J W_{i}^{T}(\lambda) J \geq 0, \quad J=\left[\begin{array}{cc}
0 & I \\
-I & 0
\end{array}\right]
\end{aligned}
$$

which depends in general nonlinearly on the spectral parameter (see [2]). Here $W_{i}(\lambda) \in$ $\mathbb{R}^{2 n \times 2 n}$ is a differentiable (hence continuous) symplectic matrix-valued function of the variable $\lambda$, such that $W_{i}(\lambda)^{T} J W_{i}(\lambda)=J$ for $i=0, \ldots, N, \lambda \in \mathbb{R}$. By [2], the matrix-function $\Psi_{i}(\lambda)$ is symmetric for all $\lambda \in \mathbb{R}$ and (1.6) describes the monotonic behavior of $W_{i}(\lambda)$ with respect to $\lambda$. In the case of spectral problem (1.1) for $y_{i}(\lambda)=\left[x_{i}^{T}(\lambda), u_{i}^{T}(\lambda)\right]^{T}, u_{i}(\lambda)=$ $\left(R_{i}(\lambda) \Delta x_{i}(\lambda)\right), i=0, \ldots, N, u_{N+1}(\lambda)=u_{N}(\lambda)+Q_{N}(\lambda) x_{N+1}(\lambda)$, the symplectic matrix in $(1.5)$ has the form

$$
W_{i}(\lambda)=\left[\begin{array}{cc}
I & R_{i}^{-1}(\lambda) \\
Q_{i}(\lambda) & I+Q_{i}(\lambda) R_{i}^{-1}(\lambda)
\end{array}\right],
$$

and the monotonicity assumption in (1.6)

$$
\begin{aligned}
\Psi_{i}(\lambda) & =\left[\begin{array}{cc}
I & -Q_{i}(\lambda) R_{i}^{-1}(\lambda) \\
0 & R_{i}^{-1}(\lambda)
\end{array}\right]\left[\begin{array}{cc}
-\frac{d}{d \lambda} Q_{i}(\lambda) & 0 \\
0 & -\frac{d}{d \lambda} R_{i}(\lambda)
\end{array}\right]\left[\begin{array}{cc}
I & 0 \\
-R_{i}^{-1}(\lambda) Q_{i}(\lambda) & R_{i}^{-1}(\lambda)
\end{array}\right] \\
& \geq 0, \quad i \in[0, N]
\end{aligned}
$$

is obviously equivalent to (1.3).

The oscillation and spectral theory for symplectic difference systems with linear dependence on the spectral parameter was successfully developed in [3-5]. For the special case of the matrix Sturm-Liouville difference equations the assumption in [3-5] on the linear dependence on $\lambda \in \mathbb{R}$ leads to the following restrictions:

$$
\begin{aligned}
& \frac{d}{d \lambda} R_{i}(\lambda) \equiv 0, \quad \frac{d}{d \lambda} \hat{R}_{i}(\lambda) \equiv 0, \quad Q_{i}(\lambda)=P_{i}-\lambda \mathcal{W}_{i}, \quad \hat{Q}_{i}(\lambda)=\hat{P}_{i}-\lambda \hat{\mathcal{W}}_{i}, \\
& P_{i}=P_{i}^{T}, \quad \hat{P}_{i}=\hat{P}_{i}, \quad \mathcal{W}_{i}=\mathcal{W}_{i}^{T}, \quad \hat{\mathcal{W}}_{i}=\hat{\mathcal{W}}_{i}^{T}, \quad \mathcal{W}_{i} \geq 0, \quad \hat{\mathcal{W}}_{i} \geq 0
\end{aligned}
$$

on the coefficient matrices $R_{i}(\lambda), \hat{R}_{i}(\lambda), Q_{i}(\lambda), \hat{Q}_{i}(\lambda)$ in (1.1), (1.2). The so-called global oscillation theorem (see $[3,4])$ applied to problem (1.1) with assumptions $(1.8)$ relates the number of finite eigenvalues of (1.1) less than or equal to a given number $\lambda=b$ to the number of focal points (counting multiplicity) of the principal solution of (1.1) with $\lambda=b$ (see [1]). Relative oscillation theory adds new aspects to the classical oscillation results by showing that matrix analogs of weighted zeros [6-10] of the Wronskian for suitable solutions of (1.1), (1.2) can be used to count the difference between the numbers of finite eigenvalues of problems (1.1), (1.2). Recall now some results of relative oscillation theory developed in $[6,7,10]$ for the scalar case of $(1.1),(1.2),(1.8)$. Consider the Sturm-Liouville 
eigenvalue problems

$$
\begin{aligned}
& \Delta\left(r_{i} \Delta x_{i}(\lambda)\right)-\left(q_{i}-\lambda\right) x_{i+1}(\lambda)=0, \quad r_{i}>0, \quad x_{0}(\lambda)=x_{N+1}(\lambda)=0, \\
& \Delta\left(\hat{r}_{i} \Delta \hat{x}_{i}(\lambda)\right)-\left(\hat{q}_{i}-\lambda\right) \hat{x}_{i+1}(\lambda)=0, \quad \hat{r}_{i}>0, \quad \hat{x}_{0}(\lambda)=\hat{x}_{N+1}(\lambda)=0, \\
& i=0, \ldots, N-1 \text {. }
\end{aligned}
$$

Introduce the Wronskian for two solutions $x_{i}, \hat{x}_{i}$ of the difference equations in (1.9), (1.10): $w_{i}(x, \hat{x})=r_{i} x_{i+1} \hat{x}_{i}-\hat{r}_{i} x_{i} \hat{x}_{i+1}$. According to [6], the Wronskian $w_{i}(x, \hat{x})$ has a node at $i$ (or a generalized zero [11, p.719] in $[i, i+1))$ if either $w_{i} w_{i+1}<0$ or $w_{i}=0, w_{i+1} \neq 0$. Then, by [6, Theorem 4.3], for problems (1.9), (1.10) with $r_{i}=\hat{r}_{i}, q_{i}=\hat{q}_{i}$ and $a<b, a, b \in \mathbb{R}$, we have

$$
\#\left(x^{(0)}(a), \hat{x}^{(N+1)}(b)\right)=\#\left\{\lambda \in \sigma_{1} \mid a<\lambda<b\right\},
$$

where $\#(x, \hat{x})$ denotes the total number of nodes of $w_{i}(x, \hat{x})$ in $(0, N+1)$, $\#\left\{\lambda \in \sigma_{1} \mid a<\lambda<b\right\}$ denotes the number of eigenvalues of (1.9) (or (1.10)) between $\lambda=a$ and $\lambda=b$ and the solutions $x_{i}^{(M)}, \hat{x}_{i}^{(M)}, M \in\{0, N+1\}$ of (1.9), (1.10) obey the conditions $x_{M}^{(M)}=0, \hat{x}_{M}^{(M)}=0$ at $i=M \in\{0, N+1\}$.

The main result in [7] extends (1.11) for the case $q_{i} \neq \hat{q}_{i}$. According to [7, Theorem 1.2], the number of weighted nodes of the Wronskian in $(0, N+1)$ equals the number of eigenvalues of (1.10) below $\lambda=b$ minus the number of eigenvalues of (1.9) below or equal to $\lambda=a$ :

$$
\#\left(x^{(0)}(a), \hat{x}^{(N+1)}(b)\right)=\#\left(x^{(N+1)}(a), \hat{x}^{(0)}(b)\right)=\#\left\{\lambda \in \sigma_{2} \mid \lambda<b\right\}-\#\left\{\lambda \in \sigma_{1} \mid \lambda \leq a\right\} .
$$

In the recent paper [10], the previous result is generalized for the case $r_{i} \neq \hat{r}_{i}, q_{i} \neq \hat{q}_{i}$. Note that relative oscillation theory for scalar spectral problems (1.1), (1.2) with nonlinear dependence on $\lambda$ (see [12]) has never been developed before.

In [13-16] we derive relative oscillation theory for symplectic difference eigenvalue problems with linear dependence on $\lambda$. Note that results in $[13,14]$ cover the special case of the matrix Sturm-Liouville eigenvalue problem (1.1), (1.8) with the linear dependence on $\lambda$ only under the additional assumption $R_{i}=\hat{R}_{i}, i=0, \ldots, N$. The relative oscillation theory which deals with the case $R_{i}(\lambda) \neq \hat{R}_{i}(\lambda)$ is called extended (see $\left.[9,10]\right)$. Results of this paper rely on the concept of finite eigenvalue of (1.5) and the global oscillation theorem which was recently proved in $[2,17]$ for symplectic eigenvalue problems $(1.5)$ with nonlinear dependence on the spectral parameter $\lambda$. We combine these results with Theorem 2.1 in [13] presenting the relation between the numbers of focal points of conjoined bases of two discrete symplectic systems with different coefficient matrices. This opens the door for generalizing relative oscillation theory for the case of spectral problems (1.1), (1.3) and (1.2), (1.4).

The paper is organized as follows. In Section 2 we recall main concepts of oscillation theory of symplectic difference systems and the comparative index theory developed in $[13,18-20]$. We introduce the relative oscillation numbers which generalize the concept of a weighted zero of the Wronskian for the matrix case. At the end of Section 2, we prove some properties of the relative oscillation numbers. 
Section 3 is devoted to relative oscillation theory for problems (1.1), (1.2). In Section 3.1 we derive the relative oscillation numbers for the pair of symplectic difference systems associated with (1.1), (1.2) (see Theorem 3.4). We also investigate other representations of the relative oscillation numbers connected with different choices of symplectic difference systems associated with (1.1), (1.2) (see Section 3.2). The main theorems (see Theorems 3.8, 3.9) proved in Section 3.3 generalize (1.12), (1.11) for the case of matrix eigenvalue problems (1.1), (1.2).

In Section 4 we provide several examples illustrating the relative oscillation theory for scalar problems (1.1), (1.2) with nonlinear dependence on the spectral parameter.

\section{Notation and auxiliary results}

We will use the following notation. For a matrix $A$, we denote by $A^{T}, A^{-1}, A^{-T}, A^{\dagger}, \operatorname{rank} A$, ind $A, A \geq 0, A \leq 0$, respectively, its transpose, inverse, transpose and inverse, MoorePenrose pseudoinverse, rank (i.e., the dimension of its image), index (i.e., the number of its negative eigenvalues), positive semidefiniteness, negative semidefiniteness. By $I$ and 0 we denote the identity and zero matrices of appropriate dimensions. We also use the notation $\prod_{k=M}^{N} A_{k}=A_{N} A_{N-1} \cdots A_{M}$ for the product of matrices $A_{N}, A_{N-1}, \ldots, A_{M}$, where we put $\prod_{k=M}^{M-1} A_{k}=I$.

Introduce the symplectic difference systems

$$
\begin{array}{ll}
y_{i+1}=W_{i} y_{i}, & W_{i}^{T} J W_{i}=J, \\
\hat{y}_{i+1}=\hat{W}_{i} \hat{y}_{i}, & \hat{W}_{i}^{T} J \hat{W}_{i}=J,
\end{array}
$$

where $y_{i} \hat{y}_{i} \in \mathbb{R}^{2 n}$.

Recall (see [11]) that $2 n \times n$ matrix solutions $Y_{i}, \hat{Y}_{i}$ of (2.1), (2.2) are said to be conjoined bases if

$$
\operatorname{rank} Y_{i}=n, \quad Y_{i}^{T} J Y_{i}=0, \quad \operatorname{rank} \hat{Y}_{i}=n, \quad \hat{Y}_{i}^{T} J \hat{Y}_{i}=0,
$$

and the conjoined bases $Y_{i}^{(M)}, \hat{Y}_{i}^{(M)}$ of (2.1), (2.2) with the initial conditions $Y_{M}^{(M)}=[0 I]^{T}$, $\hat{Y}_{M}^{(M)}=[0 I]^{T}$ at $i=M$ are said to be the principal solutions at $M$.

Note that for conjoined bases $Y_{i}, \hat{Y}_{i}$ of (2.1), (2.2) there exist symplectic fundamental matrices $Z_{i}, \hat{Z}_{i}$ such that

$$
Y_{i}=Z_{i}\left[\begin{array}{ll}
0 & I
\end{array}\right]^{T}, \quad \hat{Y}_{i}=\hat{Z}_{i}\left[\begin{array}{ll}
0 & I
\end{array}\right]^{T}
$$

(see [11, Remark 1(ii)]). Define the Wronskian

$$
w_{i}(Y, \hat{Y})=w_{i}=Y_{i}^{T} J \hat{Y}_{i}
$$

for conjoined bases of (2.1), (2.2).

Recall the definition of focal points and their multiplicities for conjoined bases of (2.1). We define the numbers of focal points of conjoined basis $Y_{i}=\left[X_{i}^{T} U_{i}^{T}\right]^{T}$ in $(i, i+1],[i, i+1)$, respectively (see [21, Definition 1], [19, Definition 3.2]): $m_{i}(Y)=\operatorname{rank} M_{i}+\operatorname{ind} P_{i}, m_{i}^{*}(Y)=$ 
$\operatorname{rank} \tilde{M}_{i}+\operatorname{ind} \tilde{P}_{i}$, where

$$
\left\{\begin{array} { l } 
{ M _ { i } = ( I - X _ { i + 1 } X _ { i + 1 } ^ { \dagger } ) B _ { i } , } \\
{ T _ { i } = I - M _ { i } ^ { \dagger } M _ { i } , } \\
{ P _ { i } = T _ { i } X _ { i } X _ { i + 1 } ^ { \dagger } B _ { i } T _ { i } , }
\end{array} \quad \left\{\begin{array}{l}
\tilde{M}_{i}=\left(I-X_{i} X_{i}^{\dagger}\right) B_{i}^{T}, \\
\tilde{T}_{i}=I-\tilde{M}_{i}^{\dagger} \tilde{M}_{i}, \\
\tilde{P}_{i}=\tilde{T}_{i} X_{i+1} X_{i}^{\dagger} B_{i}^{T} \tilde{T}_{i} .
\end{array}\right.\right.
$$

In particular, according to [1, Section 5], the number of focal points of a conjoined basis $Y_{i}=\left[X_{i}^{T}\left(R_{i}(\lambda) \Delta X_{i}\right)^{T}\right]^{T}$ of matrix Sturm-Liouville equation (1.1) in the interval $(i, i+1]$ is defined to be the number

$$
m_{i}(Y)=n-\operatorname{rank} X_{i+1}+\operatorname{ind}\left(X_{i}^{T} R_{i}(\lambda) X_{i+1}\right) .
$$

The main results of this paper are based on the comparative index theory established in [13, 18-20]. According to [19, 20], we define the comparative index for $2 n \times n$ matrices $Y$, $\hat{Y}$ with conditions (2.3) using the notation

$$
\left\{\begin{array}{l}
\mathcal{M}=\left(I-X X^{\dagger}\right) \hat{X}, \quad X=\left[\begin{array}{ll}
I & 0
\end{array}\right] Y, \quad \hat{X}=\left[\begin{array}{ll}
I & 0
\end{array}\right] \hat{Y} \\
\mathcal{T}=I-M^{\dagger} M \\
\mathcal{D}=\mathcal{D}^{T}=\mathcal{T} w^{T}(Y, \hat{Y}) X^{\dagger} \hat{X} \mathcal{T} .
\end{array}\right.
$$

The comparative index is defined by $\mu(Y, \hat{Y})=\mu_{1}(Y, \hat{Y})+\mu_{2}(Y, \hat{Y})$, where $\mu_{1}(Y, \hat{Y})=$ $\operatorname{rank} \mathcal{M}$ and $\mu_{2}(Y, \hat{Y})=\operatorname{ind} \mathcal{D}$. Introduce the dual index $\mu^{*}(Y, \hat{Y})=\mu_{1}(Y, \hat{Y})+\mu_{2}^{*}(Y, \hat{Y})$, where $\mu_{2}^{*}(Y, \hat{Y})=\operatorname{ind}(-\mathcal{D})$.

For the comparative index $\mu(Y, \hat{Y})$, we have the estimate (see Property 7 in $[19, \mathrm{p} .449]$ )

$$
\mu(Y, \hat{Y}) \leq \operatorname{rank} w(Y, \hat{Y}) \leq n,
$$

where $w(Y, \hat{Y})$ is the Wronskian given by $(2.5)$.

If $Z_{i}$ is a symplectic fundamental matrix for (2.1) connected with a conjoined basis $Y_{i}$ (see (2.4)), then, according to [19, Lemmas 3.1 and 3.2], we have

$$
\begin{aligned}
& m_{i}(Y)=\mu\left(Y_{i+1}, W_{i}\left[\begin{array}{ll}
0 & I
\end{array}\right]^{T}\right)=\mu^{*}\left(Z_{i+1}^{-1}\left[\begin{array}{ll}
0 & I
\end{array}\right]^{T}, Z_{i}^{-1}\left[\begin{array}{ll}
0 & I
\end{array}\right]^{T}\right), \\
& m_{i}^{*}(Y)=\mu^{*}\left(Y_{i}, W_{i}^{-1}\left[\begin{array}{ll}
0 & I
\end{array}\right]^{T}\right)=\mu\left(Z_{i}^{-1}\left[\begin{array}{ll}
0 & I
\end{array}\right]^{T}, Z_{i+1}^{-1}\left[\begin{array}{ll}
0 & I
\end{array}\right]^{T}\right),
\end{aligned}
$$

where $m_{i}(Y), m_{i}^{*}(Y)$ are the numbers of focal points in $(i, i+1],[i, i+1)$, respectively.

In this paper we will use the comparative index $\mu\left(\hat{Z}_{i}^{-1} Y_{i}, \hat{Z}_{i+1}^{-1} Y_{i+1}\right)$ which, according to the second formula in (2.10), presents the number of focal points $m_{i}^{*}\left(Z^{-1} \hat{Y}\right)$ in $[i, i+1)$ of the transformed conjoined basis $Z_{i}^{-1} \hat{Y}_{i}$ (see [14]) with the upper block $[I 0] Z_{i}^{-1} \hat{Y}_{i}=-w_{i}(Y, \hat{Y})$ associated with the Wronskian (2.5). To emphasize the role of the Wronskian (2.5) in the relative oscillation results, we introduce the notation $m^{*}\left(w_{i}, w_{i+1}\right):=m_{i}^{*}\left(Z^{-1} \hat{Y}\right)=$ $\mu\left(\hat{Z}_{i}^{-1} Y_{i}, \hat{Z}_{i+1}^{-1} Y_{i+1}\right)$, where by (2.7), (2.10) we have

$$
\begin{aligned}
& m^{*}\left(w_{i}, w_{i+1}\right):=\operatorname{rank} \mathcal{M}_{i}+\operatorname{ind}\left(\mathcal{P}_{i}\right), \\
& \mathcal{M}_{i}:=\left(I-w_{i}^{\dagger} w_{i}\right) w_{i+1}^{T}, \quad \mathcal{T}_{i}:=I-\mathcal{M}_{i}^{\dagger} \mathcal{M}_{i}, \quad \mathcal{P}_{i}:=\mathcal{T}_{i} w_{i+1} w_{i}^{\dagger} \mathfrak{C}_{i} \mathcal{T}_{i}, \\
& \mathfrak{C}_{i}=\left(\hat{Z}_{i}^{-1} Y_{i}\right)^{T} J\left(\hat{Z}_{i+1}^{-1} Y_{i+1}\right)=Y_{i}^{T} J \hat{W}_{i}^{-1} W_{i} Y_{i}=Y_{i+1}^{T} J W_{i} \hat{W}_{i}^{-1} Y_{i+1} .
\end{aligned}
$$


Note that according to (2.8), we have the inequality

$$
m^{*}\left(w_{i}, w_{i+1}\right) \leq \operatorname{rank}\left(\mathfrak{C}_{i}\right) \leq n
$$

because of the definition $\mathfrak{C}_{i}=\left(\hat{Z}_{i}^{-1} Y_{i}\right)^{T} J\left(\hat{Z}_{i+1}^{-1} Y_{i+1}\right)$ in (2.11).

Introduce the notation

$$
l(Y, M, N)=\sum_{i=M}^{N} m_{i}(Y), \quad l^{*}(Y, M, N)=\sum_{i=M}^{N} m_{i}^{*}(Y)
$$

for the numbers of focal points of $Y_{i}$ in $(M, N+1]$ and $[M, N+1)$.

For arbitrary $2 n \times n$ matrices $Y, \hat{Y}$ with conditions (2.3) and symplectic matrices $W, \hat{W}$, we define the operator

$$
\begin{aligned}
\mathcal{L} & (Y, \hat{Y}, W, \hat{W}) \\
& =\mu\left(\hat{W} \hat{Y}, \hat{W}\left[\begin{array}{ll}
0 & I
\end{array}\right]^{T}\right)-\mu\left(W Y, W\left[\begin{array}{ll}
0 & I
\end{array}\right]^{T}\right)+\mu(W Y, \hat{W} \hat{Y})-\mu(Y, \hat{Y}) .
\end{aligned}
$$

The main result in [19] (see Theorem 2.2) establishes the equality $\mu(W Y, W \hat{Y})=$ $\mu(Y, \hat{Y})+\mu\left(W Y, W[0 I]^{T}\right)-\mu\left(W \hat{Y}, W[0 I]^{T}\right)$ and then

$$
\mathcal{L}(Y, \hat{Y}, W, W)=0
$$

for the case $W=\hat{W}$.

When $Y:=Y_{i}, \hat{Y}:=\hat{Y}_{i}$ are conjoined bases and $W:=W_{i}, \hat{W}:=\hat{W}_{i}$ are the coefficient matrices of (2.1), (2.2), operator (2.13) takes the form

$$
\mathcal{L}\left(Y_{i}, \hat{Y}_{i}, W_{i}, \hat{W}_{i}\right)=m_{i}(\hat{Y})-m_{i}(Y)+\Delta \mu\left(Y_{i}, \hat{Y}_{i}\right)
$$

where, according to (2.9), $m_{i}(\hat{Y})=\mu\left(\hat{Y}_{i+1}, \hat{W}_{i}[0 I]^{T}\right), m_{i}(Y)=\mu\left(Y_{i+1}, W_{i}[0 I]^{T}\right)$ are the numbers of focal points in $(i, i+1]$. In [13] we derive the general representations of (2.15) in terms of the comparative index for the coefficient matrices of (2.1), (2.2). For arbitrary symplectic matrix $W$ separated into $n \times n$ blocks $A, B, C, D$ introduce the notation

$$
\langle W\rangle=\left[\begin{array}{cc}
I & 0 \\
A & B \\
0 & -I \\
C & D
\end{array}\right], \quad W=\left[\begin{array}{cc}
A & B \\
C & D
\end{array}\right]
$$

In [13, Lemma 2.3]) we prove that $4 n \times 2 n$ matrices $\langle W\rangle,\langle\hat{W}\rangle$ associated with symplectic $W, \hat{W}$ obey (2.3) (with $n$ replaced by $2 n$ ) and then the comparative index for the pair $\langle W\rangle,\langle\hat{W}\rangle$ is well defined. The results of this paper are based on the following comparison theorem proved in [13, Theorem 2.1].

Theorem 2.1 Let $Y_{i}, \hat{Y}_{i}$ be conjoined bases of (2.1), (2.2) associated with symplectic fundamental matrices $Z_{i}, \hat{Z}_{i}$ such that (2.4) hold. Then

$$
\mathcal{L}\left(Y_{i}, \hat{Y}_{i}, W_{i}, \hat{W}_{i}\right)=\#\left(Y_{i}, \hat{Y}_{i}\right)
$$


where

$$
\#\left(Y_{i}, \hat{Y}_{i}\right)=\mu\left(\left\langle\hat{Z}_{i}^{-1} Z_{i}\right\rangle,\left\langle\hat{Z}_{i+1}^{-1} Z_{i+1}\right\rangle\right)-\mu\left(\left\langle\hat{W}_{i}\right\rangle,\left\langle W_{i}\right\rangle\right),
$$

and then

$$
\begin{aligned}
\sum_{i=M}^{N} \mathcal{L}\left(Y_{i}, \hat{Y}_{i}, W_{i}, \hat{W}_{i}\right) & =l(\hat{Y}, M, N)-l(Y, M, N)+\mu\left(Y_{N+1}, \hat{Y}_{N+1}\right)-\mu\left(Y_{M}, \hat{Y}_{M}\right) \\
& =\sum_{i=M}^{N} \#\left(Y_{i}, \hat{Y}_{i}\right),
\end{aligned}
$$

where $l(\hat{Y}, M, N), l(Y, M, N)$ are the numbers of focal points in $(M, N+1]$.

The numbers $\#\left(Y_{i}, \hat{Y}_{i}\right)$ in the right-hand side of (2.16) are called the relative oscillation numbers for the symplectic difference systems (2.1), (2.2). Note that the inequality

$$
\left|\#\left(Y_{i}, \hat{Y}_{i}\right)\right| \leq \operatorname{rank}\left(W_{i}-\hat{W}_{i}\right)
$$

holds for the relative oscillation numbers in (2.16) (see the proof of Theorem 2.1 in [13]), and then $\#\left(Y_{i}, \hat{Y}_{i}\right) \equiv 0$ for the case $W_{i} \equiv \hat{W}_{i}$.

For the particular case when $Y_{i}, \hat{Y}_{i}$ are the principal solutions of (2.1), (2.2), we have the following corollary from Theorem 2.1 (see [13, Corollary 2.4]).

Corollary 2.2 Let $Y_{i}^{(0)}$ and $\hat{Y}_{i}^{(N+1)}$ be the principal solutions of (2.1) and (2.2) associated with symplectic fundamental matrices $Z_{i}^{(0)}, \hat{Z}_{i}^{(N+1)}$ such that conditions (2.4) hold. Then

$$
\begin{aligned}
l^{*}\left(\hat{Y}^{(N+1)}, 0, N\right)-l\left(Y^{(0)}, 0, N\right) & =l\left(\hat{Y}^{(0)}, 0, N\right)-l\left(Y^{(0)}, 0, N\right) \\
& =\sum_{i=0}^{N} \#\left(Y_{i}^{(0)}, \hat{Y}_{i}^{(N+1)}\right)
\end{aligned}
$$

where the relative oscillation numbers $\#\left(Y_{i}^{(0)}, \hat{Y}_{i}^{(N+1)}\right)$ are defined by $(2.17)$ for $\hat{Z}_{i}:=\hat{Z}_{i}^{(N+1)}$, $Z_{i}:=Z_{i}^{(0)}$.

Relative oscillation theory for symplectic eigenvalue problems with linear dependence on $\lambda$ developed in $[13,14]$ is based on [13, Lemma 2.2], [14, Lemma 1] where we evaluate relative oscillation numbers (2.17) assuming that the following condition

$$
W_{i} \hat{W}_{i}^{-1}=\left[\begin{array}{cc}
I & 0 \\
C_{i} & I
\end{array}\right], \quad i=0, \ldots, N
$$

holds for the matrices $W_{i}, \hat{W}_{i}$ in (2.1), (2.2) (here $C_{i}=C_{i}^{T}$ due to symplecticity of $W_{i}, \hat{W}_{i}$ ). Then, by [14, Lemma 1], we have the following representation

$$
\#\left(Y_{i}, \hat{Y}_{i}\right)=m^{*}\left(w_{i}, w_{i+1}\right)-\operatorname{ind}\left(\mathfrak{C}_{i}\right), \quad \mathfrak{C}_{i}=X_{i+1}^{T} C_{i} X_{i+1}
$$


provided (2.21) holds. In particular, for the scalar case of Sturm-Liouville equations (1.9), (1.10) with $r_{i} \equiv \hat{r}_{i}$, relative oscillation numbers (2.22) take the values \pm 1 if and only if the Wronskian $w_{i}(x, \hat{x})=r_{i} x_{i+1} \hat{x}_{i}-r_{i} x_{i} \hat{x}_{i+1}$ for solutions $x_{i}, \hat{x}_{i}$ of (1.9), (1.10) has a weighted node at $i$ according to the definition in [7] (see [14, Remark 1]).

Note that the symplectic matrices (1.7) associated with matrix Sturm-Liouville equation (1.1) for two arbitrary values $\lambda=a$ and $\lambda=b, a \neq b$ obey condition (2.21) only for the case when $R_{i}$ does not depend on $\lambda$. For two spectral problems (1.1), (1.2) condition (2.21) is satisfied under the additional assumption $R_{i} \equiv \hat{R}_{i}$. In the next section, using the special structure of symplectic matrices (1.7), we evaluate the relative oscillation numbers for problems (1.1), (1.2) for the general case $R_{i}(\lambda) \neq \hat{R}_{i}(\lambda), Q_{i}(\lambda) \neq \hat{Q}_{i}(\lambda)$. In the proofs we will use the following 'multiplicative' property of operator (2.13).

Lemma 2.3 For arbitrary symplectic matrices $S_{1}, S_{2}, \ldots, S_{p}$ and $\hat{S}_{1}, \hat{S}_{2}, \ldots, \hat{S}_{p}$, where $p \geq 1$, define $Z(r):=\prod_{k=1}^{r} S_{k}, \hat{Z}(r):=\prod_{k=1}^{r} \hat{S}_{k}, r=0,1, \ldots, p$. Then, for any $2 n \times n$ matrices $Y, \hat{Y}$ with conditions (2.3), we have

$$
\begin{aligned}
& \mathcal{L}(Y, \hat{Y}, Z(p), \hat{Z}(p))=\sum_{r=1}^{p} \mathcal{L}\left(Z(r-1) Y, \hat{Z}(r-1) \hat{Y}, S_{r}, \hat{S}_{r}\right) \\
& +\sum_{r=1}^{p}\left(\mu\left(Z(r)\left[\begin{array}{ll}
0 & I
\end{array}\right]^{T}, S_{r}\left[\begin{array}{ll}
0 & I
\end{array}\right]^{T}\right)\right. \\
& \left.-\mu\left(\hat{Z}(r)\left[\begin{array}{ll}
0 & I
\end{array}\right]^{T}, \hat{S}_{r}\left[\begin{array}{ll}
0 & I
\end{array}\right]^{T}\right)\right) .
\end{aligned}
$$

Proof By definition (2.13), in the left-hand side of (2.23) we have

$$
\begin{aligned}
\mathcal{L}(Y, \hat{Y}, Z(p), \hat{Z}(p)) \\
=\mu\left(\hat{Z}(p) \hat{Y}, \hat{Z}(p)\left[\begin{array}{ll}
0 & I
\end{array}\right]^{T}\right)-\mu\left(Z(p) Y, Z(p)\left[\begin{array}{ll}
0 & I
\end{array}\right]^{T}\right) \\
\quad+\mu(Z(p) Y, \hat{Z}(p) \hat{Y})-\mu(Y, \hat{Y}) .
\end{aligned}
$$

Similarly, for the operator $\mathcal{L}\left(Z(r-1) Y, \hat{Z}(r-1) \hat{Y}, S_{r}, \hat{S}_{r}\right)$ in the right-hand side of (2.23), we derive

$$
\begin{aligned}
& \mathcal{L}\left(Z(r-1) Y, \hat{Z}(r-1) \hat{Y}, S_{r}, \hat{S}_{r}\right) \\
& \quad=\mu\left(\hat{Z}(r) \hat{Y}, \hat{S}_{r}\left[\begin{array}{ll}
0 & I
\end{array}\right]^{T}\right)-\mu\left(Z(r) Y, S_{r}\left[\begin{array}{ll}
0 & I
\end{array}\right]^{T}\right)+\Delta \mu(Z(r-1) Y, \hat{Z}(r-1) \hat{Y}),
\end{aligned}
$$

and then

$$
\begin{aligned}
& \mathcal{L}\left(Z(r-1) Y, \hat{Z}(r-1) \hat{Y}, S_{r}, \hat{S}_{r}\right)-\mu\left(\hat{Z}(r)[0 \quad I]^{T}, \hat{S}_{r}\left[\begin{array}{ll}
0 & I
\end{array}\right]^{T}\right) \\
& +\mu\left(Z(r)\left[\begin{array}{ll}
0 & I
\end{array}\right]^{T}, S_{r}\left[\begin{array}{ll}
0 & I
\end{array}\right]^{T}\right) \\
& =-\mathcal{L}\left(\hat{Z}(r-1) \hat{Y}, \hat{Z}(r-1)\left[\begin{array}{ll}
0 & I
\end{array}\right]^{T}, \hat{S}_{r}, \hat{S}_{r}\right)+\Delta \mu\left(\hat{Z}(r-1) \hat{Y}, \hat{Z}(r-1)\left[\begin{array}{ll}
0 & I
\end{array}\right]^{T}\right) \\
& +\mathcal{L}\left(Z(r-1) Y, Z(r-1)[0 \quad I]^{T}, S_{r}, S_{r}\right)-\Delta \mu\left(Z(r-1) Y, Z(r-1)\left[\begin{array}{ll}
0 & I
\end{array}\right]^{T}\right) \\
& +\Delta \mu(Z(r-1) Y, \hat{Z}(r-1) \hat{Y}) \text {. }
\end{aligned}
$$


By (2.14), $\mathcal{L}\left(\hat{Z}(r-1) \hat{Y}, \hat{Z}(r-1)[0 I]^{T}, \hat{S}_{r}, \hat{S}_{r}\right)=\mathcal{L}\left(Z(r-1) Y, Z(r-1)[0 I]^{T}, S_{r}, S_{r}\right)=0$, then summing (2.25) from $r=1$ to $r=p$, we derive (2.24). The proof is completed.

\section{Remark 2.4}

(i) Assume that $Y_{i}, \hat{Y}_{i}$ are conjoined bases and $Y_{i}^{(M)}, \hat{Y}_{i}^{(M)}$ are the principal solutions of (2.1), (2.2) at $M \in \mathbb{Z}$. If we apply Lemma 2.3 for the case $p=N-M+1, N \geq M$,

$$
Y:=Y_{M}, \quad \hat{Y}:=\hat{Y}_{M}, \quad S_{k}:=W_{k-1+M}, \quad \hat{S}_{k}:=\hat{W}_{k-1+M}, \quad k=1, \ldots, p,
$$

then $Z(r) Y:=Y_{M+r}, \hat{Z}(r) \hat{Y}:=Y_{M+r}$, and $Z(r)[0 I]^{T}:=Y_{M+r}^{(M)}, \hat{Z}(r)[0 I]^{T}:=\hat{Y}_{M+r}^{(M)}$, $r=0, \ldots, N-M+1$. For the given case, equality (2.23) takes the form

$$
\begin{aligned}
& \mathcal{L}\left(Y_{M}, \hat{Y}_{M}, \prod_{i=M}^{N} W_{i}, \prod_{i=M}^{N} \hat{W}_{i}\right) \\
& \quad=\sum_{i=M}^{N} \mathcal{L}\left(Y_{i}, \hat{Y}_{i}, W_{i}, \hat{W}_{i}\right)+l\left(Y^{(M)}, M, N\right)-l\left(\hat{Y}^{(M)}, M, N\right) .
\end{aligned}
$$

In particular, if systems $(2.1),(2.2)$ are disconjugate in $[M, N]$, i.e. $l\left(Y^{(M)}, M, N\right)=l\left(\hat{Y}^{(M)}, M, N\right)=0$, then

$$
\mathcal{L}\left(Y_{M}, \hat{Y}_{M}, \prod_{i=M}^{N} W_{i}, \prod_{i=M}^{N} \hat{W}_{i}\right)=\sum_{i=M}^{N} \mathcal{L}\left(Y_{i}, \hat{Y}_{i}, W_{i}, \hat{W}_{i}\right)
$$

(ii) Note that (2.26) gives us possibility to replace pointwise evaluation of $N-M+1$ operators (2.15) associated with the pairs $W_{i}, \hat{W}_{i}$ by computation of only one operator (2.13) associated with the products $\prod_{i=M}^{N} W_{i}, \prod_{i=M}^{N} \hat{W}_{i}$. In this paper we apply Lemma 2.3 in the opposite direction. Assume that for any $i$ the following factorizations $W_{i}=\prod_{k=1}^{p} S_{k}, \hat{W}_{i}=\prod_{k=1}^{p} \hat{S}_{k}$ hold for the coefficient matrices of (2.1), (2.2) (here $p$ and $S_{k}, \hat{S}_{k}$ can depend on $i$ ). Then for $Y:=Y_{i}, \hat{Y}:=\hat{Y}_{i}$ we have $Z(p) Y:=Y_{i+1}, \hat{Z}(p) \hat{Y}:=Y_{i+1}$ and Lemma 2.3 presents the action of operator (2.15) at the point $i$ as a result of actions of $p$ operators associated with the factors $S_{k}, \hat{S}_{k}$, $k=1, \ldots, p$ (see the proofs of Lemma 3.3 and Theorem 3.4 in the next section).

We will also need the following result which is based on (2.22). It is well known (see $[11,20,22])$ that symplectic transformations with lower block triangular matrices do not change the number of focal points. In particular, if we introduce the symplectic matrices

$$
K_{i}=\left[\begin{array}{cc}
I & 0 \\
\mathcal{P}_{i} & I
\end{array}\right], \quad \hat{K}_{i}=\left[\begin{array}{cc}
I & 0 \\
\hat{\mathcal{P}}_{i} & I
\end{array}\right], \quad \mathcal{P}_{i}=\mathcal{P}_{i}^{T}, \quad \hat{\mathcal{P}}_{i}=\hat{\mathcal{P}}_{i}^{T},
$$

then $m\left(Y_{i}\right)=m\left(K_{i} Y_{i}\right), m\left(\hat{Y}_{i}\right)=m\left(\hat{K}_{i} \hat{Y}_{i}\right)$, and for operator (2.15) we have

$$
\begin{aligned}
& \mathcal{L}\left(K_{i} Y_{i}, \hat{K}_{i} \hat{Y}_{i}, K_{i+1} W_{i} K_{i}^{-1}, \hat{K}_{i+1} \hat{W}_{i} \hat{K}_{i}^{-1}\right) \\
& \quad=\hat{m}\left(Y_{i}\right)-m\left(Y_{i}\right)+\Delta \mu\left(K_{i} Y_{i}, \hat{K}_{i} \hat{Y}_{i}\right) \\
& \quad=\mathcal{L}\left(Y_{i}, \hat{Y}_{i}, W_{i}, \hat{W}_{i}\right)+\Delta f_{i}, \quad f_{i}=\mu\left(K_{i} Y_{i}, \hat{K}_{i} \hat{Y}_{i}\right)-\mu\left(Y_{i}, \hat{Y}_{i}\right) .
\end{aligned}
$$


Note that we can rewrite $f_{i}$ in the form $f_{i}=\mathcal{L}\left(Y_{i}, \hat{Y}_{i}, K_{i}, \hat{K}_{i}\right)$, where $K_{i}, \hat{K}_{i}$ obey assumption (2.21). Then for $f_{i}$ we have the representation

$$
f_{i}=m^{*}\left(w_{i}, \tilde{w}_{i}\right)-\operatorname{ind}\left(\mathfrak{C}_{i}\right), \quad \mathfrak{C}_{i}=X_{i}^{T}\left(\mathcal{P}_{i}-\hat{\mathcal{P}}_{i}\right) X_{i}, \tilde{w}_{i}=Y_{i}^{T} K_{i}^{T} J \hat{K}_{i} \hat{Y}_{i}
$$

where the Wronskian $w_{i}$ is given by $(2.5)$ and $m^{*}\left(w_{i}, \tilde{w}_{i}\right)$ is defined by $(2.11)$ with $w_{i+1}:=\tilde{w}_{i}$. So we have proved the following lemma.

Lemma 2.5 The relative oscillation numbers $\#\left(Y_{i}, \hat{Y}_{i}\right)$ and $\#\left(K_{i} Y_{i}, \hat{K}_{i} \hat{Y}_{i}\right)$ with $K_{i}, \hat{K}_{i}$ given by (2.27) are connected by the equation

$$
\#\left(Y_{i}, \hat{Y}_{i}\right)=\#\left(K_{i} Y_{i}, \hat{K}_{i} \hat{Y}_{i}\right)-\Delta f_{i},
$$

where $f_{i}$ is presented by (2.29). In particular, for the case $Y_{i}:=Y_{i}^{(0)}$ and $\hat{Y}_{i}:=\hat{Y}_{i}^{(N+1)}$, we have $f_{N+1}=f_{0}=0$, and then, by Corollary 2.2,

$$
l\left(\hat{Y}^{(0)}, 0, N\right)-l\left(Y^{(0)}, 0, N\right)=\sum_{i=0}^{N} \#\left(Y_{i}^{(0)}, \hat{Y}_{i}^{(N+1)}\right)=\sum_{i=0}^{N} \#\left(K_{i} Y_{i}^{(0)}, \hat{K}_{i} \hat{Y}_{i}^{(N+1)}\right) .
$$

\section{Relative oscillation theory for matrix Sturm-Liouville difference equations}

\subsection{Relative oscillation numbers for matrix Sturm-Liouville difference equations}

Consider the evaluation of relative oscillation numbers (2.17) for the special case of the matrix Sturm-Liouville equations. Introduce the conjoined bases of systems (2.1), (2.2) associated with (1.1), (1.2):

$$
\begin{aligned}
& Y_{i}(\lambda)=\left[\begin{array}{ll}
X_{i}^{T}(\lambda) & U_{i}^{T}(\lambda)
\end{array}\right]^{T}, \quad \hat{Y}_{i}(\lambda)=\left[\begin{array}{ll}
\hat{X}_{i}^{T}(\lambda) & \hat{U}_{i}^{T}(\lambda)
\end{array}\right]^{T}, \\
& U_{i}(\lambda)=R_{i}(\lambda) \Delta X_{i}(\lambda), \quad \hat{U}_{i}(\lambda)=\hat{R}_{i}(\lambda) \Delta \hat{X}_{i}(\lambda), \quad i=0, \ldots, N, \\
& U_{N+1}(\lambda)=U_{N}(\lambda)+Q_{N}(\lambda) X_{N+1}(\lambda), \quad \hat{U}_{N+1}(\lambda)=\hat{U}_{N}(\lambda)+\hat{Q}_{N}(\lambda) \hat{X}_{N+1}(\lambda) .
\end{aligned}
$$

Note that the coefficients $Q_{N}(\lambda), \hat{Q}_{N}(\lambda)$ are not needed in equations (1.1), (1.2), but for convenience we define them at $i=N$ such that (1.3), (1.4) hold. In Remark 3.2 we will show that the results of this section do not depend on the definition of $Q_{N}(\lambda), \hat{Q}_{N}(\lambda)$.

The Wronskian (2.5) of $Y_{i}(a), \hat{Y}_{i}(b)$ for two fixed values $\lambda=a$ and $\lambda=b$

$$
\begin{aligned}
w_{i}(X(a), \hat{X}(b)) & =w_{i}(a, b) \\
& =X_{i}^{T}(a) \hat{U}_{i}(b)-U_{i}^{T}(a) \hat{X}_{i}(b), \quad a, b \in \mathbb{R}, i=0, \ldots, N+1
\end{aligned}
$$

obeys the equation

$$
\begin{aligned}
\Delta w_{i}(a, b)= & -\left(\Delta\left(X_{i}^{T}(a)\right)\left(R_{i}(a)-R_{i}(b)\right) \Delta \hat{X}_{i}(b)\right. \\
& \left.+X_{i+1}^{T}(a)\left(Q_{i}(a)-\hat{Q}_{i}(b)\right) \hat{X}_{i+1}(b)\right), \quad i=0, \ldots, N .
\end{aligned}
$$


The symplectic coefficient matrices (1.7) associated with (1.1), (1.2) can be presented in the special factorized form

$$
\begin{aligned}
& W_{i}(\lambda)=\left[\begin{array}{cc}
I & 0 \\
Q_{i}(\lambda) & I
\end{array}\right]\left[\begin{array}{cc}
I & R_{i}^{-1}(\lambda) \\
0 & I
\end{array}\right]=L_{i}(\lambda) H_{i}(\lambda), \\
& \hat{W}_{i}(\lambda)=\left[\begin{array}{cc}
I & 0 \\
\hat{Q}_{i}(\lambda) & I
\end{array}\right]\left[\begin{array}{cc}
I & \hat{R}_{i}^{-1}(\lambda) \\
0 & I
\end{array}\right]=\hat{L}_{i}(\lambda) \hat{H}_{i}(\lambda) .
\end{aligned}
$$

The main result of this section is based on the consideration of the following particular cases.

Lemma 3.1 (Case I) Assume that for spectral problems (1.1), (1.3) and (1.2), (1.4), the matrices $R_{i}(\lambda), \hat{R}_{i}(\lambda)$ obey the conditions

$$
\frac{d}{d \lambda} R_{i}(\lambda)=\frac{d}{d \lambda} \hat{R}_{i}(\lambda)=0, \quad R_{i} \equiv \hat{R}_{i}, \quad i=0,1, \ldots, N,
$$

then relative oscillation numbers (2.17) have the form

$$
\begin{aligned}
& \#_{I}\left(Y_{i}(a), \hat{Y}_{i}(b)\right)=m^{*}\left(w_{i}(a, b), w_{i+1}(a, b)\right)-\operatorname{ind}\left(\mathfrak{C}_{i}(a, b)\right), \\
& \mathfrak{C}_{i}(a, b)=X_{i+1}^{T}(a)\left(Q_{i}(a)-\hat{Q}_{i}(b)\right) X_{i+1}(a),
\end{aligned}
$$

where $m^{*}\left(w_{i}(a, b), w_{i+1}(a, b)\right)$ is given by $(2.11)$ with $\mathfrak{C}_{i}:=\mathfrak{C}_{i}(a, b)$ and $w_{i}:=w_{i}(a, b)$ defined by (3.2).

Proof For case (3.5) we have that condition (2.21) is obviously satisfied for matrices (3.4):

$$
W_{i}(a) \hat{W}_{i}^{-1}(b)=L_{i}(a) \hat{L}_{i}(b)=\left[\begin{array}{cc}
I & 0 \\
Q_{i}(a)-\hat{Q}_{i}(b) & I
\end{array}\right]
$$

and then applying (2.22) we derive (3.6).

Remark 3.2 Note that in the definition of symplectic systems (1.7), (3.4) we can put $Q_{N}(\lambda)=\hat{Q}_{N}(\lambda)=0$ and then $\#_{I}\left(Y_{N}(a), \hat{Y}_{N}(b)\right)=0$. However, for the case when $\hat{Y}_{i}:=$ $\hat{Y}_{i}^{(N+1)}$, we have $\#_{I}\left(Y_{N}(a), \hat{Y}_{N}(b)\right)=0$ for any choice of $Q_{N}(\lambda), \hat{Q}_{N}(\lambda)$. Indeed, for this case $w_{N}(a, b)=w_{N+1}(a, b)=X_{N+1}^{T}(a)$ by (3.3), (3.2) and according to (2.11), we have $m^{*}\left(w_{N}(a, b), w_{N+1}(a, b)\right)=\operatorname{ind}\left(\mathfrak{C}_{N}(a, b)\right)$.

Lemma 3.3 (Case II) Assume that for spectral problems (1.1), (1.3) and (1.2), (1.4) the matrices $Q_{i}(\lambda), \hat{Q}_{i}(\lambda)$ obey the conditions

$$
\frac{d}{d \lambda} Q_{i}(\lambda)=\frac{d}{d \lambda} \hat{Q}_{i}(\lambda)=0, \quad Q_{i} \equiv \hat{Q}_{i}, \quad i=0,1, \ldots, N
$$

then relative oscillation numbers (2.17) have the form

$$
\begin{aligned}
& \#_{I I}\left(Y_{i}(a), \hat{Y}_{i}(b)\right)=m^{*}\left(w_{i}(a, b), w_{i+1}(a, b)\right)-\operatorname{ind}\left(\mathfrak{B}_{i}(a, b)\right)+P_{i}, \\
& \mathfrak{B}_{i}(a, b)=U_{i}^{T}(a)\left(\hat{R}_{i}^{-1}(b)-R_{i}^{-1}(a)\right) U_{i}(a), \quad U_{i}(\lambda)=R_{i}(\lambda) \Delta X_{i}(\lambda) .
\end{aligned}
$$


Here $m^{*}\left(w_{i}(a, b), w_{i+1}(a, b)\right)$ is defined by $(2.11)$ where $\mathfrak{C}_{i}:=\mathfrak{B}_{i}(a, b)$, and the number $P_{i}$ does not depend on $a, b$ :

$$
P_{i}=\operatorname{ind}\left(\hat{R}_{i}\left(\lambda_{0}\right)\right)-\operatorname{ind}\left(R_{i}\left(\lambda_{0}\right)\right), \quad \lambda_{0} \in \mathbb{R} .
$$

Proof Note that for case (3.8) matrices (3.4) obey the condition

$$
\hat{W}_{i}^{-1}(b) W_{i}(a)=\hat{H}_{i}^{-1}(b) H_{i}(a)=\left[\begin{array}{cc}
I & R_{i}^{-1}(a)-\hat{R}_{i}^{-1}(b) \\
0 & I
\end{array}\right] .
$$

The symplectic upper block triangular factors $H_{i}(a), \hat{H}_{i}(b)$ in (3.11) can be represented in the form

$$
H_{i}(a)=J^{T} K_{i}(a) J, \quad \hat{H}_{i}(b)=J^{T} \hat{K}_{i}(b) J,
$$

where $K_{i}(a), \hat{K}_{i}(b)$ are the symplectic lower block triangular matrices. Assumption (3.8) implies that $L_{i}(a) \equiv \hat{L}_{i}(b)=L_{i}$ in (3.4). Consider operator (2.15) for case (3.8). Applying Lemma 2.3 for $p=4, S_{1}=\hat{S}_{1}:=J, S_{2}:=K_{i}(a), \hat{S}_{2}:=\hat{K}_{i}(b), S_{3}=\hat{S}_{3}:=J^{T}, S_{4}=\hat{S}_{4}=L_{i}, Y:=Y_{i}$, $\hat{Y}:=\hat{Y}_{i}$, we have

$$
\begin{aligned}
& \mathcal{L}\left(Y_{i}, \hat{Y}_{i}, W_{i}(a), \hat{W}_{i}(b)\right)=\mathcal{L}\left(Y_{i}, \hat{Y}_{i}, L_{i} J^{T} K_{i}(a) J, L_{i} J^{T} \hat{K}_{i}(b) J\right) \\
& =\mathcal{L}\left(Y_{i}, \hat{Y}_{i}, J, J\right)+\mathcal{L}\left(J Y_{i}, J \hat{Y}_{i}, K_{i}(a), \hat{K}_{i}(b)\right) \\
& +\mathcal{L}\left(K_{i}(a) J Y_{i}, \hat{K}_{i}(b) J \hat{Y}_{i}, J^{T}, J^{T}\right) \\
& +\mathcal{L}\left(H_{i}(a) Y_{i}(a), \hat{H}_{i}(b) \hat{Y}_{i}(b), L_{i}, L_{i}\right) \\
& +\left\{\mu\left(H_{i}(a)\left[\begin{array}{ll}
0 & I
\end{array}\right]^{T}, J^{T}\left[\begin{array}{ll}
0 & I
\end{array}\right]^{T}\right)\right. \\
& \left.-\mu\left(\hat{H}_{i}(b)\left[\begin{array}{ll}
0 & I
\end{array}\right]^{T}, J^{T}\left[\begin{array}{ll}
0 & I
\end{array}\right]^{T}\right)\right\},
\end{aligned}
$$

where the addends in the braces correspond to the last sum in (2.23). Taking into account that the right-hand side of operator (2.13) equals zero for $W=\hat{W}$ (see (2.14)) and evaluating the difference $\left\{\mu\left(H_{i}(a)[0 I]^{T}, J^{T}[0 I]^{T}\right)-\mu\left(\hat{H}_{i}(b)[0 I]^{T}, J^{T}[0 I]^{T}\right)\right\}$ according to the definition of the comparative index, we have

$$
\mathcal{L}\left(Y_{i}(a), \hat{Y}_{i}(b), W_{i}(a), \hat{W}_{i}(b)\right)=\mathcal{L}\left(J Y_{i}(a), J \hat{Y}_{i}(b), K_{i}(a), \hat{K}_{i}(b)\right)+\operatorname{ind}\left(\hat{R}_{i}(b)\right)-\operatorname{ind}\left(R_{i}(a)\right) .
$$

Recall that the symmetric nonsingular matrices $R_{i}(\lambda), \hat{R}_{i}(\lambda)$ are continuous functions in $\lambda$ and then their eigenvalues have the constant sign for $\lambda \in \mathbb{R}$. So we have $\operatorname{ind}\left(\hat{R}_{i}(a)\right)=$ $\operatorname{ind}\left(\hat{R}_{i}\left(\lambda_{0}\right)\right), \operatorname{ind}\left(R_{i}(a)\right)=\operatorname{ind}\left(R_{i}\left(\lambda_{0}\right)\right)$ for any $\lambda_{0} \in \mathbb{R}$.

Note that the symplectic matrices $K_{i}(a), \hat{K}_{i}(b)$ in the operator $\mathcal{L}\left(J Y_{i}, f \hat{Y}_{i}, K_{i}(a), \hat{K}_{i}(b)\right)$ are unit lower block triangular and then they obey condition (2.21):

$$
K_{i}(a) \hat{K}_{i}^{-1}(b)=J H_{i}(a) \hat{H}_{i}^{-1}(b) J^{T}=\left[\begin{array}{cc}
I & 0 \\
\hat{R}_{i}^{-1}(b)-R_{i}^{-1}(a) & I
\end{array}\right] .
$$


Evaluating $\mathcal{L}\left(J Y_{i}(a), J \hat{Y}_{i}(b), K_{i}(a), \hat{K}_{i}(b)\right)$ according to (2.22), where $\mathfrak{C}_{i}$ should be replaced by $\mathfrak{B}_{i}(a, b)=U_{i}^{T}(a)\left(\hat{R}_{i}^{-1}(b)-R_{i}^{-1}(a)\right) U_{i}(a)$, we derive (3.9) with $P_{i}$ given by (3.10). The proof is completed.

Consider the evaluation of the relative oscillation numbers for the general case. Introduce the following Wronskian:

$$
w_{i^{*}}(a, b)=X_{i+1}^{T}(a) \hat{U}_{i}(b)-U_{i}^{T}(a) \hat{X}_{i+1}(b), \quad i^{*} \in(i, i+1)
$$

for $U_{i}(\lambda), \hat{U}_{i}(\lambda)$ defined as in (3.1). Here we use the intermediate point $i^{*} \in(i, i+1)$ for the convenient interpretation of subsequent results. Note that

$$
\begin{aligned}
& w_{i^{*}}(a, b)=w_{i}(a, b)-U_{i}^{T}(a)\left(\hat{R}_{i}^{-1}(b)-R_{i}^{-1}(a)\right) \hat{U}_{i}(b), \\
& w_{i+1}(a, b)=w_{i^{*}}(a, b)-X_{i+1}^{T}(a)\left(Q_{i}(a)-\hat{Q}_{i}(b)\right) \hat{X}_{i+1}(b),
\end{aligned}
$$

and then summing (3.13), (3.14) we derive (3.3). In particular, if case I takes place (i.e., conditions (3.5) hold), we have $w_{i^{*}}(a, b)=w_{i}(a, b)$ by (3.13) and similarly, by $(3.14), w_{i+1}(a, b)=$ $w_{i^{*}}(a, b)$ in case II.

Theorem 3.4 (General case) For spectral problems (1.1), (1.3) and (1.2), (1.4) associated with symplectic matrices (3.4), relative oscillation numbers (2.17) have the form

$$
\#\left(Y_{i}(a), \hat{Y}_{i}(b), W_{i}(a), \hat{W}_{i}(b)\right)=\#_{I I}\left(i, i^{*}\right)+\#_{I}\left(i^{*}, i+1\right),
$$

where the numbers

$$
\begin{aligned}
& \#_{I I}\left(i, i^{*}\right)=m^{*}\left(w_{i}(a, b), w_{i^{*}}(a, b)\right)-\operatorname{ind}\left(\mathfrak{B}_{i}(a, b)\right)+P_{i}, \\
& \#_{I}\left(i^{*}, i+1\right)=m^{*}\left(w_{i^{*}}(a, b), w_{i+1}(a, b)\right)-\operatorname{ind}\left(\mathfrak{C}_{i}(a, b)\right)
\end{aligned}
$$

are evaluated according to (3.9), (3.10) and (3.6), respectively, with $w_{i+1}(a, b):=w_{i^{*}}(a, b)$ for case II and $w_{i}(a, b):=w_{i^{*}}(a, b)$ for case I.

Proof For the proof we use factorizations (3.4) and Lemma 2.3. Putting in Lemma 2.3 $p=2, S_{1}:=H_{i}(a), \hat{S}_{1}:=\hat{H}_{i}(b), S_{2}:=L_{i}(a), \hat{S}_{2}:=\hat{L}_{i}(b), Y:=Y_{i}, \hat{Y}:=\hat{Y}_{i}$, we derive

$$
\begin{aligned}
\mathcal{L} & \left(Y_{i}(a), \hat{Y}_{i}(b), W_{i}(a), \hat{W}_{i}(b)\right) \\
\quad= & \mathcal{L}\left(Y_{i}(a), \hat{Y}_{i}(b), L_{i}(a) H_{i}(a), \hat{L}_{i}(b) \hat{H}_{i}(b)\right) \\
\quad= & \mathcal{L}\left(Y_{i}(a), \hat{Y}_{i}(b), H_{i}(a), \hat{H}_{i}(b)\right)+\mathcal{L}\left(H_{i}(a) Y_{i}(a), \hat{H}_{i}(b) \hat{Y}_{i}(b), L_{i}(a), \hat{L}_{i}(b)\right) .
\end{aligned}
$$

By (3.11), (3.7) operators $\mathcal{L}\left(Y_{i}(a), \hat{Y}_{i}(b), H_{i}(a), \hat{H}_{i}(b)\right)$ and $\mathcal{L}\left(H_{i}(a) Y_{i}(a), \hat{H}_{i}(b) \hat{Y}_{i}(b), L_{i}(a)\right.$, $\left.\hat{L}_{i}(b)\right)$ can be evaluated according to cases II and I, respectively. For case II, we have that the conjoined bases $Y_{i}(a), \hat{Y}_{i}(b)$ obey the symplectic systems $Y_{i^{*}}(a)=H_{i}(a) Y_{i}(a), \hat{Y}_{i^{*}}(b)=$ $\hat{H}_{i}(b) \hat{Y}_{i}(b), i^{*} \in(i, i+1)$, and then we have to use the Wronskian $Y_{i^{*}}(a)^{T} J \hat{Y}_{i^{*}}(b)=w_{i^{*}}(a, b)$ given by (3.12) instead of $w_{i+1}(a, b)$. Similarly, in case I we use that $Y_{i^{*}}(a), \hat{Y}_{i^{*}}(b)$ obey the symplectic systems $Y_{i+1}(a)=L_{i}(a) Y_{i^{*}}(a), \hat{Y}_{i+1}(b)=\hat{L}_{i}(b) \hat{Y}_{i^{*}}(b)$ and then we apply (3.6) replacing $w_{i}(a, b)$ by $w_{i^{*}}(a, b)$. Finally, we point out that such modifications of (3.9) and (3.6) 
do not touch the matrices $\mathfrak{B}_{i}(a, b), \mathfrak{C}_{i}(a, b)$ according to their definitions in (3.9) and (3.6). The proof is completed.

Now we formulate some properties of the relative oscillation numbers given by (3.15), (3.16), (3.17).

\section{Proposition 3.5}

(i) If case I takes place (i.e., conditions (3.5) hold), then in (3.15) we have $\#\left(Y_{i}(a), \hat{Y}_{i}(b)\right)=\#_{I}\left(Y_{i}(a), \hat{Y}_{i}(b)\right)$ for $\#_{I}\left(Y_{i}(a), \hat{Y}_{i}(b)\right)$ given by (3.6). Similarly, for case II, \# $\left(Y_{i}(a), \hat{Y}_{i}(b)\right)=\#_{I I}\left(Y_{i}(a), \hat{Y}_{i}(b)\right)$ with $\#_{I I}\left(Y_{i}(a), \hat{Y}_{i}(b)\right)$ given by (3.9).

(ii) If the conditions

$$
Q_{i}(a) \geq \hat{Q}_{i}(b), \quad R_{i}(a) \geq \hat{R}_{i}(b)
$$

hold, then the relative oscillation numbers given by (3.15), (3.16), (3.17) are nonnegative. In particular, for the case $Q_{i}(\lambda) \equiv \hat{Q}_{i}(\lambda), R_{i}(\lambda) \equiv \hat{R}_{i}(\lambda), a<b$, the relative oscillation numbers are presented in the form

$$
\#\left(Y_{i}(a), Y_{i}(b)\right)=m^{*}\left(w_{i}, w_{i^{*}}\right)+m^{*}\left(w_{i^{*}}, w_{i+1}\right) \geq 0, \quad a<b,
$$

where $m^{*}\left(w_{i}, w_{i^{*}}\right)$ and $m^{*}\left(w_{i^{*}}, w_{i+1}\right)$ are defined by $(2.11)$ with $\mathfrak{C}_{i}:=\mathfrak{B}_{i}(a, b) \geq 0$ and $\mathfrak{C}_{i}:=\mathfrak{C}_{i}(a, b) \geq 0$ given by (3.9) and (3.6), respectively.

(iii) For the relative oscillation numbers in (3.15), we have the estimate

$$
\left|\#\left(Y_{i}(a), \hat{Y}_{i}(b)\right)\right| \leq \operatorname{rank}\left(R_{i}(a)-\hat{R}_{i}(b)\right)+\operatorname{rank}\left(Q_{i}(a)-\hat{Q}_{i}(b)\right) \leq 2 n .
$$

Proof For the proof of (i), we use (3.13), (3.14). Case I implies (see (3.13)) that $w_{i}(a, b)=$ $w_{i^{*}}(a, b)$ and the matrices $\mathfrak{B}_{i}(a, b), P_{i}$ in (3.9), (3.10) equal zero. Then $\#_{I I}\left(i, i^{*}\right)=0$ and $\#\left(Y_{i}(a), \hat{Y}_{i}(b)\right)=\#_{I}\left(Y_{i}(a), \hat{Y}_{i}(b)\right)$. In a similar way, for case II, $\mathfrak{C}_{i}(a, b)=0$, and we get from (3.14) that $w_{i+1}(a, b)=w_{i^{*}}(a, b)$. Finally, it follows that $\#_{I}\left(i^{*}, i+1\right)=0$, \#( $\left.Y_{i}(a), \hat{Y}_{i}(b)\right)=$ $\#_{I I}\left(Y_{i}(a), \hat{Y}_{i}(b)\right)$.

For the proof of (ii) we note that under assumptions (3.18) numbers (3.17) are nonnegative because of $\operatorname{ind}\left(\mathfrak{C}_{i}(a, b)\right)=0$. For the proof $\#_{I I}\left(i, i^{*}\right) \geq 0$, we use the following index result:

$$
\operatorname{ind} \hat{R}_{i}(b)-\operatorname{ind} R_{i}(a)=\operatorname{ind}\left(\hat{R}_{i}^{-1}(b)-R_{i}^{-1}(a)\right)-\operatorname{ind}\left(R_{i}(a)-\hat{R}_{i}(b)\right)
$$

which follows from (2.14) for the case $Y:=\left[R_{i}(a) I\right]^{T}, \hat{Y}:=\left[\hat{R}_{i}(b) I\right]^{T}, W:=J$. Recall that in the proof of Lemma 3.9 we used ind $R_{i}(\lambda)=\operatorname{ind} R_{i}\left(\lambda_{0}\right)$, ind $\hat{R}_{i}(\lambda)=\operatorname{ind} \hat{R}_{i}\left(\lambda_{0}\right)$ according to monotonicity assumptions (1.3), (1.4). Then, by (3.21), ind $\hat{R}_{i}(b)-\operatorname{ind} R_{i}(a)=P_{i}=$ $\operatorname{ind}\left(\hat{R}_{i}^{-1}(b)-R_{i}^{-1}(a)\right)$, where we use that $\operatorname{ind}\left(R_{i}(a)-\hat{R}_{i}(b)\right)=0$. Finally, we have $\#_{I I}\left(i, i^{*}\right)=$ $\left(P_{i}-\operatorname{ind}\left(\mathfrak{B}_{i}(a, b)\right)\right)+m^{*}\left(w_{i}, w_{i^{*}}\right) \geq 0$ because of

$$
P_{i}-\operatorname{ind}\left(\mathfrak{B}_{i}(a, b)\right)=\operatorname{ind}\left(\hat{R}_{i}^{-1}(b)-R_{i}^{-1}(a)\right)-\operatorname{ind}\left(U_{i}^{T}(a)\left(\hat{R}_{i}^{-1}(b)-R_{i}^{-1}(a)\right) U_{i}(a)\right) \geq 0 .
$$

For the case $Q_{i}(\lambda) \equiv \hat{Q}_{i}(\lambda), R_{i}(\lambda) \equiv \hat{R}_{i}(\lambda), a \leq b$, we additionally have $P_{i}=\operatorname{ind}\left(R_{i}^{-1}(b)-\right.$ $\left.R_{i}^{-1}(a)\right)=0$ and in $(3.16)$ ind $\left(\mathfrak{B}_{i}(a, b)\right)=0$. Then the proof of (ii) is completed. 
By (2.19), relative oscillation numbers (3.16), (3.17) obey the inequalities

$$
\begin{aligned}
& \left|\#_{I I}\left(i, i^{*}\right)\right| \leq \operatorname{rank}\left(H_{i}(a)-\hat{H}_{i}(b)\right)=\operatorname{rank}\left(R_{i}(a)-\hat{R}_{i}(b)\right), \\
& \left|\#_{I}\left(i^{*}, i+1\right)\right| \leq \operatorname{rank}\left(L_{i}(a)-\hat{L}_{i}(b)\right)=\operatorname{rank}\left(Q_{i}(a)-\hat{Q}_{i}(b)\right),
\end{aligned}
$$

and then for the relative oscillation numbers in (3.15) we have estimate (3.20). The proof is completed.

\subsection{Other representations of the relative oscillation numbers for matrix Sturm-Liouville difference equations}

By expanding of the operators $\Delta$, the matrix Sturm-Liouville equation in (1.1) (or (1.2)) can be rewritten as the three-term recurrence $[1,23]$

$$
\begin{aligned}
& R_{i+1}(\lambda) x_{i+2}-T_{i}(\lambda) x_{i+1}+R_{i}(\lambda) x_{i}=0, \quad i \in[0, N-1], \\
& T_{i}(\lambda)=R_{i+1}(\lambda)+R_{i}(\lambda)+Q_{i}(\lambda) .
\end{aligned}
$$

In [1, Theorem 3.1] the following result for equation (3.22) is proved. If we put

$$
\begin{aligned}
& \tilde{y}_{i}=\left[\begin{array}{ll}
x_{i}^{T} & u_{i}^{T}
\end{array}\right]^{T}, \quad u_{i}:=R_{i}(\lambda) x_{i+1}-P_{i}(\lambda) x_{i}, \quad i \in[0, N], \\
& u_{N+1}=\left(T_{N}(\lambda)-P_{N+1}(\lambda)\right) x_{N+1}-R_{N}(\lambda) x_{N}
\end{aligned}
$$

with an arbitrary symmetric matrix $P_{i}(\lambda)$, then, according to [1, Theorem 3.1], $\tilde{y}_{i}$ solves the symplectic difference system with coefficients depending on $P_{i}(\lambda)$. Here we assume (see [1]) that $R_{N+1}(\lambda), Q_{N}(\lambda)$ are defined so that conditions (1.3) hold.

The choice $P_{i}(\lambda):=R_{i}(\lambda), i \in[0, N+1]$ leads to the symplectic system with matrix (1.7). The solution $y_{i}(\lambda)$ of this system is connected with $\tilde{y}_{i}$ in (3.23) by the following symplectic transformation:

$$
\tilde{y}_{i}(\lambda)=K_{i}(\lambda) y_{i}(\lambda), \quad K_{i}(\lambda)=\left[\begin{array}{cc}
I & 0 \\
R_{i}(\lambda)-P_{i}(\lambda) & I
\end{array}\right] .
$$

The Wronskian $\tilde{w}_{i}(X(a), \hat{X}(b))$ for conjoined bases of the transformed systems also depends on the choice of $P_{i}(\lambda), \hat{P}_{i}(\lambda)$. So we have

$$
w_{i}(X(a), \hat{X}(b))=\tilde{w}_{i}(X(a), \hat{X}(b))+X_{i}^{T}(a)\left(R_{i}(a)-\hat{R}_{i}(b)-P_{i}(a)+\hat{P}_{i}(b)\right) \hat{X}_{i}(b),
$$

where $w_{i}(X(a), \hat{X}(b))$ is given by (3.2).

In particular, the Wronskian in [10] (for the scalar problems (1.9), (1.10))

$$
\tilde{w}_{i}(X(a), \hat{X}(b))=\tilde{w}_{i}(a, b)=X_{i}^{T}(a) \hat{R}_{i}(b) \hat{X}_{i+1}(b)-X_{i+1}^{T}(a) R_{i}(a) \hat{X}_{i}(b)
$$

corresponds to the choice $P_{i}(\lambda):=0, \hat{P}_{i}:=0$ in (3.23). Transformation (3.24) in this case leads to the following coefficients matrices:

$$
\tilde{W}_{i}(\lambda)=\left[\begin{array}{cc}
0 & R_{i}^{-1}(\lambda) \\
-R_{i}(\lambda) & T_{i}(\lambda) R_{i}^{-1}(\lambda)
\end{array}\right], \quad \breve{W}_{i}(\lambda)=\left[\begin{array}{cc}
0 & \hat{R}_{i}^{-1}(\lambda) \\
-\hat{R}_{i}(\lambda) & \hat{T}_{i}(\lambda) \hat{R}_{i}^{-1}(\lambda)
\end{array}\right],
$$


where $T_{i}(\lambda)$ is defined in (3.22) and $\hat{T}_{i}(\lambda)$ is defined similarly for (1.2). Formula (3.25) for $P_{i}(\lambda):=0, \hat{P}_{i}:=0$ takes the form

$$
w_{i}(X(a), \hat{X}(b))=\tilde{w}_{i}(X(a), \hat{X}(b))+X_{i}^{T}(a)\left(R_{i}(a)-\hat{R}_{i}(b)\right) \hat{X}_{i}(b) .
$$

It is easy to see that

$$
\begin{aligned}
& \tilde{W}_{i}(a) \breve{W}_{i}^{-1}(b)=\tilde{L}_{i}(a, b), \\
& \tilde{L}_{i}(a, b)=\left[\begin{array}{cc}
M_{i}(a, b) & 0 \\
T_{i}(a) M_{i}(a, b)-M_{i}^{-T}(a, b) \hat{T}_{i}(b) & M_{i}^{-T}(a, b)
\end{array}\right], \\
& M_{i}(a, b)=R_{i}^{-1}(a) \hat{R}_{i}(b),
\end{aligned}
$$

where $M_{i}(a, b)=I$ for the case $R_{i}(a)=\hat{R}_{i}(b)$. Slightly modifying the proof of Lemma 1 in [14, p.1233] (see also [13, Lemma 2.1]), we have the following representation of the relative oscillation numbers for the transformed systems with matrices (3.27):

$$
\#\left(\tilde{Y}_{i}(a), \breve{Y}_{i}(b)\right)=m^{*}\left(\tilde{w}_{i}(a, b), \tilde{w}_{i+1}(a, b)\right)-\mu\left(\tilde{Y}_{i+1}(a), \tilde{L}_{i}(a, b) \tilde{Y}_{i+1}(a)\right)
$$

Here $\tilde{Y}_{i}(a)=K_{i}(a) Y_{i}(a), \breve{Y}_{i}(b)=\hat{K}_{i}(b) \hat{Y}_{i}(b)$ and $\tilde{w}_{i}(a, b)$ is given by (3.26). The number $m^{*}\left(\tilde{w}_{i}(a, b), \tilde{w}_{i+1}(a, b)\right)$ is defined by $(2.11)$ with $\mathfrak{C}_{i}:=\mathcal{D}_{i}(a, b)$, where

$$
\begin{aligned}
\mathcal{D}_{i}(a, b)= & X_{i+1}^{T}(a) R_{i}(a) \hat{R}_{i}^{-1}(b)\left(T_{i}(a)-\hat{T}_{i}(b)\right) X_{i+1}(a)+X_{i}^{T}(a) \hat{R}_{i}(b) X_{i+1}(a) \\
& -X_{i+1}^{T}(a) R_{i}(a) \hat{R}_{i}^{-1}(b) R_{i}(a) X_{i}(a) .
\end{aligned}
$$

In particular, for case I (see Lemma 3.1), relative oscillation numbers (3.30) coincide with $\#_{I}\left(Y_{i}(a), Y_{i}(b)\right)$ given by (3.6). In the general case, by Lemma 2.5 , relative oscillation numbers (3.30) are connected with (3.15) by the formula

$$
\#\left(Y_{i}(a), Y_{i}(b)\right)=\#\left(\tilde{Y}_{i}(a), \breve{Y}_{i}(b)\right)-\Delta f_{i}
$$

where $f_{i}$ is given by (2.29) with $\mathfrak{C}_{i}:=X_{i}^{T}(a)\left(R_{i}(a)-\hat{R}_{i}(b)\right) X_{i}(a)$. For relative oscillation numbers (3.30), we have the following estimate (compare with (3.20)):

$$
\left|\#\left(\tilde{Y}_{i}(a), \breve{Y}_{i}(b)\right)\right| \leq \operatorname{rank} \mathcal{D}_{i}(a, b) \leq n,
$$

with $\mathcal{D}_{i}(a, b)$ given by (3.31). For the proof, we apply inequalities $(2.8),(2.12)$ to the addends in the right-hand side of (3.30) such that $m^{*}\left(\tilde{w}_{i}(a, b), \tilde{w}_{i+1}(a, b)\right) \leq \operatorname{rank} \mathcal{D}_{i}(a, b)$, $\mu\left(\tilde{Y}_{i+1}(a), \tilde{L}_{i}(a, b) \tilde{Y}_{i+1}(a)\right) \leq \operatorname{rank} \mathcal{D}_{i}(a, b)$. By analogy with Remark 3.2, we can also show that \# $\left(\tilde{Y}_{N}(a), \breve{Y}_{N}^{(N+1)}(b)\right)=0$ for any choice of $Q_{N}(\lambda), R_{N+1}(\lambda), \hat{Q}_{N}(\lambda), \hat{R}_{N+1}(\lambda)$.

However, we cannot guarantee that Proposition 3.5(ii) holds for relative oscillation numbers (3.30). In particular, for the scalar case of problems (1.1), (1.2), we show that (3.30) takes the value -1 for the case $Q_{i}(\lambda) \equiv \hat{Q}_{i}(\lambda), R_{i}(\lambda) \equiv \hat{R}_{i}(\lambda), a<b$ under the monotonicity assumptions in (1.3), (1.4) (see Example 4.2 in Section 4). Moreover, one can verify by 
direct computations that the monotonicity assumption (1.6) holds for (3.27) only if $R_{i}(\lambda)$ does not depend on $\lambda$. So we have

$$
\Psi_{i}(\lambda)=J\left[\begin{array}{cc}
R_{i}^{-1}(\lambda) & 0 \\
T_{i}(\lambda) R_{i}^{-1}(\lambda) & I
\end{array}\right]\left[\begin{array}{cc}
0 & \frac{d}{d \lambda} R_{i}(\lambda) \\
\frac{d}{d \lambda} R_{i}(\lambda) & -\frac{d}{d \lambda} T_{i}(\lambda)
\end{array}\right]\left[\begin{array}{cc}
R_{i}^{-1}(\lambda) & R_{i}^{-1}(\lambda) T_{i}(\lambda) \\
0 & I
\end{array}\right] J^{T} \geq 0,
$$

and the last condition is equivalent to

$$
\frac{d}{d \lambda} R_{i}(\lambda)=0, \quad \frac{d}{d \lambda} Q_{i}(\lambda) \leq 0
$$

where we use Lemma 2.7 in [22] to evaluate the index of a symmetric matrix with zero diagonal block (see also index results in [24]).

\subsection{Relative oscillation theorems}

In this section we prove analogs of (1.12), (1.11) for the case of matrix eigenvalue problems (1.1), (1.2). Recall the notion of the finite eigenvalue introduced for (1.5) in [2].

Definition 3.6 Let $Y_{i}^{(M)}(\lambda)=\left[X_{i}(\lambda)^{T} U_{i}(\lambda)^{T}\right]^{T}$ be the principal solution of (1.5) at $M=0$. The number $\lambda_{k} \in \mathbb{R}$ is a finite eigenvalue of (1.5) if

$$
\theta\left(\lambda_{k}\right):=\operatorname{rank} X_{N+1}\left(\lambda_{k}{ }^{-}\right)-\operatorname{rank} X_{N+1}\left(\lambda_{k}\right) \geq 1,
$$

where $\operatorname{rank} X_{N+1}\left(\lambda_{k}{ }^{-}\right)=\lim _{\lambda \rightarrow \lambda_{k}-0} \operatorname{rank} X_{N+1}(\lambda)$ and $\theta\left(\lambda_{k}\right)$ is the multiplicity of $\lambda_{k}$.

The global oscillation theorem in [17] connects the number of the finite eigenvalues (including their multiplicities) of (1.5) with the number of focal points of the principal solution under the additional assumption $\operatorname{Im} B_{i}(\lambda)=$ const, $\lambda \in \mathbb{R}$, where $B_{i}(\lambda)$ is the block of $W_{i}(\lambda)$ in the upper right corner (see [17, Theorem 3.2]). The symplectic matrix (1.7) satisfies this condition, and then we can formulate the global oscillation theorem for the special case of problem (1.1).

Theorem 3.7 Assume (1.1), (1.3). Then the finite eigenvalues of (1.1) are isolated, bounded from below, and there exists $p \in\{0,1, \ldots, n N\}$ such that for any $b \in \mathbb{R}$

$$
l\left(Y^{(0)}(b), 0, N\right)=\#\left\{\lambda \in \sigma_{1} \mid \lambda \leq b\right\}+p,
$$

where $\#\left\{\lambda \in \sigma_{1} \mid \lambda \leq b\right\}$ is the number of finite eigenvalues of $(1.1)$ in $(-\infty, b], l\left(Y^{(0)}(b), 0, N\right)=$ $\sum_{i=0}^{N} m_{i}\left(Y^{(0)}(b)\right)$ is the number of focal points (2.6) of the principal solution $Y_{i}^{(0)}(\lambda)$ in $(0, N+1]$ for $\lambda=b$, and

$$
p=l\left(Y^{(0)}\left(\lambda_{0}\right), 0, N\right), \quad \lambda_{0}<\min \left\{\lambda \in \sigma_{1}\right\} .
$$

Using Corollary 2.2 and the connection (3.34) between the number of focal points of the principal solution $l\left(Y^{(0)}(b), 0, N\right)$ and the number of finite eigenvalues we can easily prove the following main theorems.

Theorem 3.8 (Relative oscillation theorem for matrix Sturm-Liouville equations) Let $\sigma_{1}$, $\sigma_{2}$ be the finite spectra and $Y_{i}^{(0)}(\lambda), \hat{Y}_{i}^{(N+1)}(\lambda)$ be the principal solutions of $(1.1),(1.3)$ and 
(1.2), (1.4). Then there exists the constant $\mathfrak{P} \in\{0, \pm 1, \ldots, \pm n N\}$ such that for all $a, b \in \mathbb{R}$, the following identity holds:

$$
\#\left\{\lambda \in \sigma_{2} \mid \lambda \leq b\right\}-\#\left\{\lambda \in \sigma_{1} \mid \lambda \leq a\right\}=\sum_{i=0}^{N} \#\left(Y_{i}^{(0)}(a), \hat{Y}_{i}^{(N+1)}(b)\right)-\mathfrak{P} .
$$

Here the relative oscillation numbers are defined by (3.15), (3.16), (3.17),

$$
\mathfrak{P}=\sum_{i=0}^{N} \#\left(Y_{i}^{(0)}\left(\lambda_{0}\right), \hat{Y}_{i}^{(N+1)}\left(\lambda_{0}\right)\right)=p_{2}-p_{1},
$$

and $p_{2}=l\left(\hat{Y}^{(0)}\left(\lambda_{0}\right), 0, N+1\right), p_{1}=l\left(Y^{(0)}\left(\lambda_{0}\right), 0, N+1\right), \lambda_{0}<\min \left\{\lambda \in \sigma_{1} \cup \sigma_{2}\right\}$.

Proof According to Theorem 3.7,

$$
\#\left\{\lambda \in \sigma_{1} \mid \lambda \leq a\right\}=l\left(Y^{(0)}(a), 0, N\right)-p_{1}, \quad \#\left\{\lambda \in \sigma_{2} \mid \lambda \leq b\right\}=l\left(\hat{Y}^{(0)}(b), 0, N\right)-p_{2},
$$

where, by (3.35) $p_{2}-p_{1}=l\left(\hat{Y}^{(0)}\left(\lambda_{0}\right), 0, N\right)-l\left(Y^{(0)}\left(\lambda_{0}\right), 0, N\right), \lambda_{0}<\min \left\{\lambda \in \sigma_{1} \cup \sigma_{2}\right\}$. Then

$$
\#\left\{\lambda \in \sigma_{2} \mid \lambda \leq b\right\}-\#\left\{\lambda \in \sigma_{1} \mid \lambda \leq a\right\}=l\left(\hat{Y}^{(0)}(b), 0, N\right)-l\left(Y^{(0)}(a), 0, N\right)-\mathfrak{P} .
$$

By Corollary 2.2, we derive

$$
\begin{aligned}
& l\left(\hat{Y}^{(0)}(b), 0, N\right)-l\left(Y^{(0)}(a), 0, N\right)=\sum_{i=0}^{N} \#\left(Y_{i}^{(0)}(a), \hat{Y}_{i}^{(N+1)}(b)\right), \\
& p_{2}-p_{1}=\mathfrak{P}=\sum_{i=0}^{N} \#\left(Y_{i}^{(0)}\left(\lambda_{0}\right), \hat{Y}_{i}^{(N+1)}\left(\lambda_{0}\right)\right), \quad \lambda_{0}<\min \left\{\lambda \in \sigma_{1} \cup \sigma_{2}\right\}
\end{aligned}
$$

for \#( $\left.Y_{i}^{(0)}(a), \hat{Y}_{i}^{(N+1)}(b)\right), a, b \in \mathbb{R}$ given by (3.15), (3.16), (3.17). Substituting the last representations into (3.37), we complete the proof of (3.36).

For the case $R_{i}(\lambda) \equiv \hat{R}_{i}(\lambda), Q_{i}(\lambda) \equiv \hat{Q}_{i}(\lambda), b>a$, Theorem 3.8 presents the number of finite eigenvalues of $(1.1)$ in $(a, b]$.

Theorem 3.9 (Renormalized oscillation theorem) For problem (1.1), (1.3) for $a<b$, the following identity holds:

$$
\begin{aligned}
& \#\{\lambda \in \sigma \mid a<\lambda \leq b\}=\sum_{i=0}^{N} \#\left(Y_{i}^{(0)}(a), Y_{i}^{(N+1)}(b)\right), \\
& \#\left(Y_{i}^{(0)}(a), Y_{i}^{(N+1)}(b)\right)=m^{*}\left(w_{i}, w_{i^{*}}\right)+m^{*}\left(w_{i^{*}}, w_{i+1}\right) \geq 0,
\end{aligned}
$$

and $m^{*}\left(w_{i}, w_{i^{*}}\right), m^{*}\left(w_{i^{*}}, w_{i+1}\right)$ are defined by $(2.11)$ with $\mathfrak{C}_{i}:=\mathfrak{B}_{i}(a, b) \geq 0$, and $\mathfrak{C}_{i}:=$ $\mathfrak{C}_{i}(a, b) \geq 0$ given by (3.9) and (3.6), respectively.

Proof For the case $R_{i}(\lambda) \equiv \hat{R}_{i}(\lambda), Q_{i}(\lambda) \equiv \hat{Q}_{i}(\lambda), b>a$, we have in (3.36) that $\#\{\lambda \in \sigma \mid \lambda \leq$ $b\}-\#\{\lambda \in \sigma \mid \lambda \leq a\}=\#\{\lambda \in \sigma \mid a<\lambda \leq b\}$ and $\mathfrak{P}=p_{1}-p_{2}=0$. Applying Proposition 3.5(ii), we complete the proof of Theorem 3.9. 


\section{Remark 3.10}

(i) In the definition of (3.16), we use the number $P_{i}$ given by (3.10) which does not depend on $a, b$. Then it makes sense to introduce the new constant

$$
\begin{aligned}
\tilde{\mathfrak{P}} & =\mathfrak{P}-\sum_{i=0}^{N} P_{i}=\sum_{i=0}^{N} \#\left(Y_{i}^{(0)}\left(\lambda_{0}\right), \hat{Y}_{i}^{(N+1)}\left(\lambda_{0}\right)\right)-\sum_{i=0}^{N}\left(\operatorname{ind}\left(\hat{R}_{i}\left(\lambda_{0}\right)\right)-\operatorname{ind}\left(R_{i}\left(\lambda_{0}\right)\right)\right), \\
& \lambda_{0}<\min \left\{\lambda \in \sigma_{1} \cup \sigma_{2}\right\}
\end{aligned}
$$

and use identity (3.36) in the form

$$
\#\left\{\lambda \in \sigma_{2} \mid \lambda \leq b\right\}-\#\left\{\lambda \in \sigma_{1} \mid \lambda \leq a\right\}=\sum_{i=0}^{N}\left\{\#\left(Y_{i}^{(0)}(a), \hat{Y}_{i}^{(N+1)}(b)\right)-P_{i}\right\}-\tilde{\mathfrak{P}} .
$$

For the numbers $\#\left(Y_{i}^{(0)}(a), \hat{Y}_{i}^{(N+1)}(b)\right)-P_{i}$, we can also improve the estimate (3.20)

$$
\left|\#\left(Y_{i}^{(0)}(a), \hat{Y}_{i}^{(N+1)}(b)\right)-P_{i}\right| \leq \operatorname{rank} \mathfrak{B}_{i}(a, b)+\operatorname{rank} \mathfrak{C}_{i}(a, b)
$$

for $\mathfrak{B}_{i}(a, b), \mathfrak{C}_{i}(a, b)$ given by (3.9) and (3.6). Indeed, by analogy with the proof of (3.33), we have

$$
\begin{aligned}
\left|\#_{I I}\left(Y_{i}^{(0)}(a), \hat{Y}_{i}^{(N+1)}(b)\right)-P_{i}\right| & =\left|m^{*}\left(w_{i}(a, b), w_{i^{*}}(a, b)\right)-\operatorname{ind}\left(\mathfrak{B}_{i}(a, b)\right)\right| \\
& \leq \operatorname{rank} \mathfrak{B}_{i}(a, b),
\end{aligned}
$$

where we use that $m^{*}\left(w_{i}(a, b), w_{i^{*}}(a, b)\right) \leq \operatorname{rank} \mathfrak{B}_{i}(a, b)$ by $(2.12)$ and $\operatorname{ind}\left(\mathfrak{B}_{i}(a, b)\right) \leq \operatorname{rank} \mathfrak{B}_{i}(a, b)$. Similarly, we can prove that $\left|\#_{I}\left(Y_{i}^{(0)}(a), \hat{Y}_{i}^{(N+1)}(b)\right)\right| \leq \operatorname{rank} \mathfrak{C}_{i}(a, b)$. Note that (3.36) and (3.40) coincide for the case $P_{i}=\operatorname{ind}\left(R_{i}(\lambda)\right)-\operatorname{ind}\left(\hat{R}_{i}(\lambda)\right)=0$, for example, under the traditional assumption $R_{i}(\lambda)>0, \hat{R}_{i}(\lambda)>0$.

(ii) According to Lemma 2.5, in the right-hand sides of (3.36), (3.38), we can use other representations of the relative oscillation numbers investigated in Section 3.2. In particular, we can use the relative oscillation numbers given by (3.30).

(iii) Note that Theorem 2.1 presents the connection between the numbers of focal points of conjoined bases of two arbitrary symplectic systems. In particular, we can apply Theorem 2.1 to the general case of two symplectic eigenvalue problems (1.5) with nonlinear dependence on the spectral parameter (see $[2,17])$. The main properties of relative oscillation numbers (2.16) for this general case are subject of the present investigation of the author.

\section{Examples}

This section is devoted to examples which illustrate the applications of Theorems 3.8, 3.9 to the scalar spectral problems (1.1), (1.2). Note that the classical oscillation theory for scalar spectral problems (1.1) with nonlinear dependence on the spectral parameter is developed in [12]. 
Example 4.1 Consider problem (1.1) for the scalar Sturm-Liouville difference equation

$$
\begin{aligned}
& \Delta\left(r_{i}(\lambda) \Delta x_{i}(\lambda)\right)-q_{i}(\lambda) x_{i+1}(\lambda)=0, \quad i=0,1,2,3, \\
& x_{0}(\lambda)=x_{4}(\lambda)=0, \\
& r_{i}(\lambda)=(-1)^{i+1} \exp \left((-1)^{i} \lambda\right), \quad q_{i}(\lambda)=-\lambda,
\end{aligned}
$$

then for the principal solution of (4.1) at 0 , defined by the initial conditions $x_{0}(\lambda)=0$, $x_{1}(\lambda)=1 / r_{0}(\lambda)$, we have

$$
x_{4}(\lambda)=-\lambda^{3}-6 \lambda^{2} \sinh (\lambda)-8 \lambda \sinh ^{2}(\lambda)+2 \lambda+4 \sinh (\lambda)
$$

and the finite eigenvalues of (4.1) are the zeros of $x_{4}(\lambda): \lambda_{1} \approx-0.6167186, \lambda_{2}=0, \lambda_{3} \approx$ 0.6167186 .

According to Theorem 3.9, we have \# $\left(Y_{i}^{(0)}(a), Y_{i}^{(N+1)}(b)\right)=m^{*}\left(w_{i}, w_{i^{*}}\right)+m^{*}\left(w_{i^{*}}, w_{i+1}\right)$, where for the scalar case

$$
\begin{aligned}
& m^{*}\left(w_{i}, w_{i^{*}}\right)= \begin{cases}1, & w_{i^{*}} \neq 0, w_{i} w_{i^{*}} \leq 0 \\
0, & \text { otherwise }\end{cases} \\
& m^{*}\left(w_{i^{*}}, w_{i+1}\right)= \begin{cases}1, & w_{i+1} \neq 0, w_{i+1} w_{i^{*}} \leq 0 \\
0, & \text { otherwise }\end{cases}
\end{aligned}
$$

Then, according to (4.2) and Theorem 3.9, the number of finite eigenvalues of problem (4.1) in the interval $(a, b]$ equals the total number of generalized zeros of the Wronskian in all intervals $\left[i, i^{*}\right),\left[i^{*}, i+1\right), i=0, \ldots, N, i^{*} \in(i, i+1)$. For example, if $a=-0.8, b=1.8$, we have the three sign changes of the Wronskian $w_{i}(a, b)$ (see Figure 1), then according to Theorem 3.9, the three eigenvalues of problem (4.1): $\lambda_{1} \approx-0.6167186, \lambda_{2}=0$, $\lambda_{3} \approx 0.6167186$ are located in $(-0.8,1.8]$. Note that the relative oscillation number $0 \leq$ $\#\left(Y_{i}^{(0)}(a), Y_{i}^{(N+1)}(b)\right) \leq 2$ achieves its maximal value 2 at the point $i=2$.

Example 4.2 In this example we evaluate the number of eigenvalues of problem (4.1) in Example 4.1 using the representation of the relative oscillation numbers in the form of (3.30). Recall that (3.30) are associated with the Wronskian $\tilde{w}_{i}(a, b)$ given by (3.26). For the scalar case representation, (3.30) can be simplified as follows:

$$
\begin{aligned}
& \#\left(\tilde{Y}_{i}(a), \tilde{Y}_{i}(b)\right)=m^{*}\left(\tilde{w}_{i}(a, b), \tilde{w}_{i+1}(a, b)\right)-\operatorname{ind}\left(\mathcal{D}_{i}(a, b)\right), \\
& \mathcal{D}_{i}(a, b)=r_{i}(a) / r_{i}(b)\left(T_{i}(a)-T_{i}(b)\right) x_{i+1}(a)^{2}+1 / r_{i}(b)\left(r_{i}(b)^{2}-r_{i}(a)^{2}\right) x_{i}(a) x_{i+1}(a), \\
& T_{i}(\lambda)=r_{i}(\lambda)+r_{i+1}(\lambda)+q_{i}(\lambda) .
\end{aligned}
$$

Then we have

$$
\#\left(\tilde{Y}_{i}(a), \tilde{Y}_{i}(b)\right)= \begin{cases}1, & \mathcal{D}_{i}(a, b)>0, \tilde{w}_{i+1} \neq 0, \tilde{w}_{i} \tilde{w}_{i^{*}} \leq 0 \\ -1, & \mathcal{D}_{i}(a, b)<0, \tilde{w}_{i} \neq 0, \tilde{w}_{i} \tilde{w}_{i+1} \leq 0 \\ 0, & \text { otherwise. }\end{cases}
$$



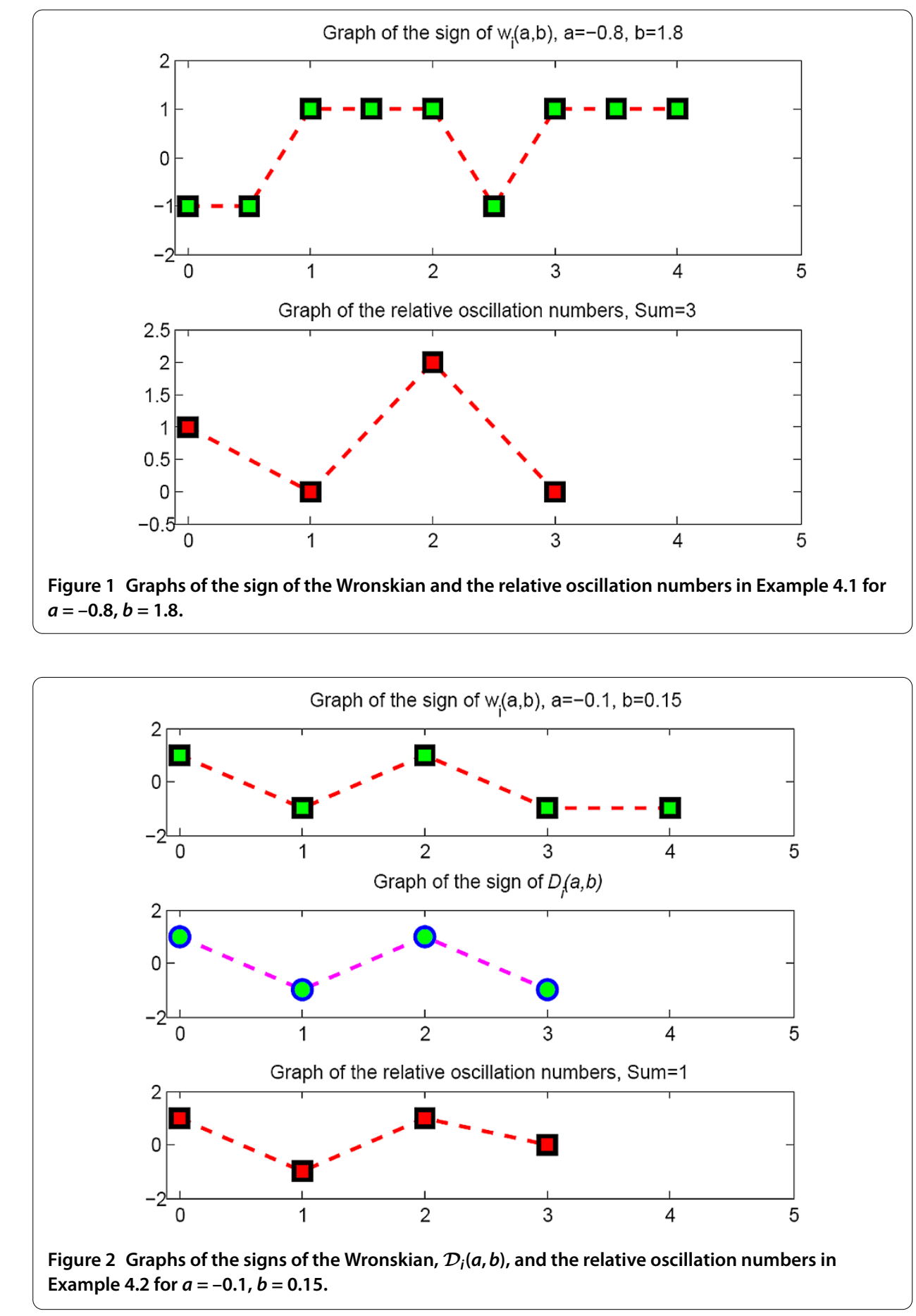

It is possible to show that definition (4.3) is equivalent to the definition of a weighted node of the Wronskian in [10]. According to Remark 3.10(ii), the number of finite eigenvalues of problem (4.1) in the interval $(a, b]$ equals the total number of weighted nodes of the Wronskian in $[0, N]$. For example (see Figure 2), for $a=-0.1, b=0.15$, the total number of weighted nodes of the Wronskian in the interval $[0,3]$ equals 1 , and we have only one eigenvalue $\lambda_{2} \in(-0.1,0.15]$. Note that the relative oscillation number $-1 \leq$ $\#\left(\tilde{Y}_{i}^{(0)}(a), \tilde{Y}_{i}^{(N+1)}(b)\right) \leq 1$ achieves its minimal value -1 at the point $i=1$. 


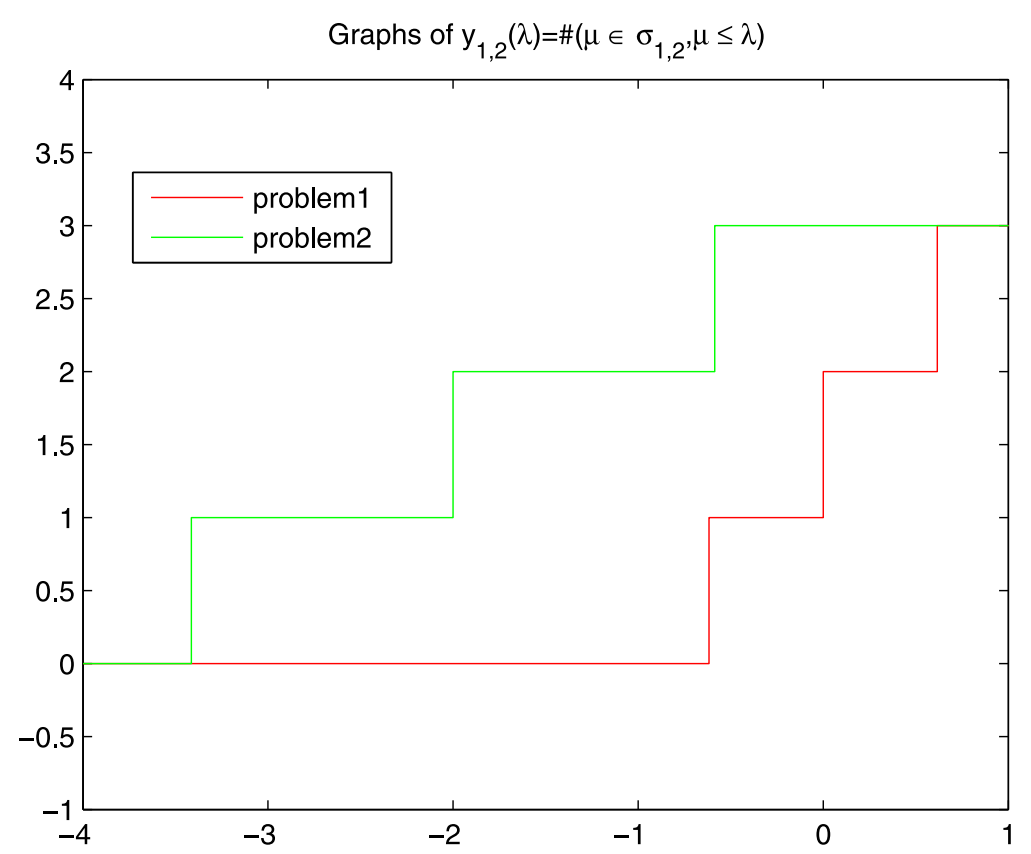

Figure 3 Graphs of the functions $y_{1,2}(\lambda)$ of the number of eigenvalues below or equal to $\lambda, \lambda \in \mathbb{R}$ for problems 1, 2 in Example 4.3.

Example 4.3 Consider spectral problem (4.1) (problem1) and the following spectral problem (problem2):

$$
\begin{aligned}
& \Delta\left(\hat{r}_{i}(\lambda) \Delta \hat{x}_{i}(\lambda)\right)-\hat{q}_{i}(\lambda) \hat{x}_{i+1}(\lambda)=0, \quad i=0,1,2,3, \\
& \hat{x}_{0}(\lambda)=\hat{x}_{4}(\lambda)=0, \\
& \hat{r}_{i}(\lambda)=(-1)^{i}, \quad \hat{q}_{i}(\lambda)=-(\lambda+2) .
\end{aligned}
$$

The finite eigenvalues of (4.4) are the zeros of the equation

$$
\begin{aligned}
& \hat{x}_{4}(\lambda)=(\lambda+2)^{3}-2 \lambda-4=0, \quad \hat{\lambda}_{1}=-2^{1 / 2}-2 \approx-3.4142136, \\
& \hat{\lambda}_{2}=-2, \quad \hat{\lambda}_{3}=2^{1 / 2}-2 \approx-0.5857864 .
\end{aligned}
$$

The localization of the eigenvalues of (4.1) and (4.4) is shown in Figure 3 where we present the functions $y_{1,2}(\lambda)$ of the number of eigenvalues below or equal to $\lambda, \lambda \in \mathbb{R}$.

According to Theorem 3.8, we can calculate the difference between the numbers of eigenvalues of (4.4) and (4.1) using the relative oscillation numbers \# $\left(Y_{i}^{(0)}(a), \hat{Y}_{i}^{(N+1)}(b)\right)=$ $\#_{I I}\left(i, i^{*}\right)+\#_{I}\left(i^{*}, i+1\right)$. For the scalar case, we have

$$
\#_{I I}\left(i, i^{*}\right)-P_{i}= \begin{cases}1, & \hat{r}_{i}^{-1}(b)-r_{i}^{-1}(a)>0 \text { and } w_{i^{*}} \neq 0, w_{i} w_{i^{*}} \leq 0 \\ -1, & \hat{r}_{i}^{-1}(b)-r_{i}^{-1}(a)<0 \text { and } w_{i} \neq 0, w_{i} w_{i^{*}} \leq 0 \\ 0, & \text { otherwise }\end{cases}
$$

where the number $P_{i}$ given by (3.10) is defined as $P_{i}=\operatorname{ind}\left(\hat{r}_{i}\left(\lambda_{0}\right)\right)-\operatorname{ind}\left(r_{i}\left(\lambda_{0}\right)\right)=(-1)^{i+1}$. Then we can say that the Wronskian has a weighted node at $i$ if $\#_{I I}\left(i, i^{*}\right)-P_{i}= \pm 1$. Accord- 


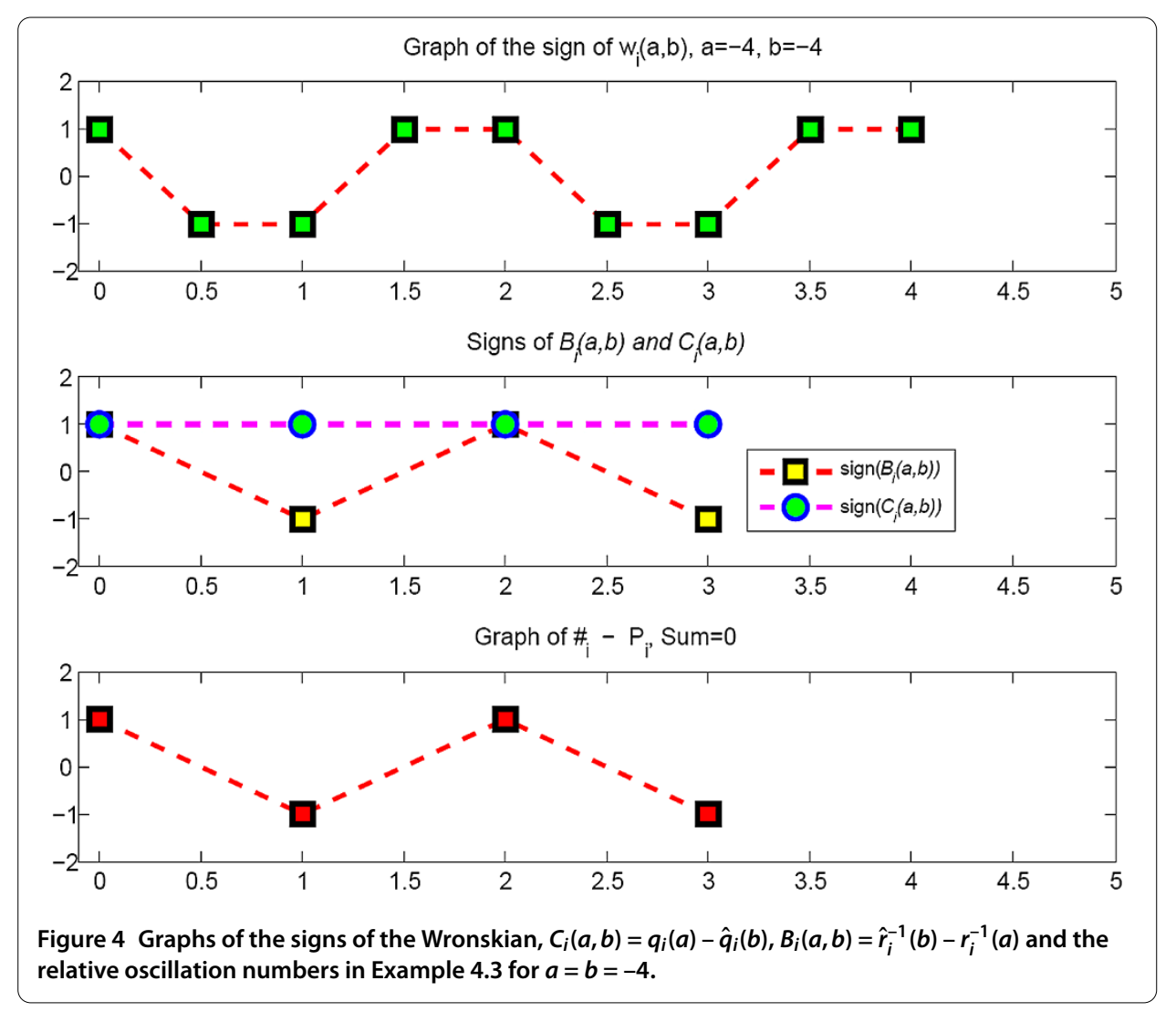

ing to Remark 3.10(i), we can consider the sum $\sum_{i=0}^{3} P_{i}=0$ as the parameter of problem (4.4).

Similarly, for the scalar case, we derive

$$
\#_{I}\left(i^{*}, i+1\right)= \begin{cases}1, & q_{i}(a)-\hat{q}_{i}(b)>0 \text { and } w_{i+1} \neq 0, w_{i+1} w_{i^{*}} \leq 0 \\ -1, & q_{i}(a)-\hat{q}_{i}(b)<0 \text { and } w_{i^{*}} \neq 0, w_{i+1} w_{i^{*}} \leq 0 \\ 0, & \text { otherwise }\end{cases}
$$

and say that the Wronskian has a weighted node at $i^{*}$ if $\#_{I}\left(i^{*}, i+1\right)= \pm 1$.

Denote $C_{i}(a, b)=q_{i}(a)-\hat{q}_{i}(b), B_{i}(a, b)=\hat{r}_{i}^{-1}(b)-r_{i}^{-1}(a)$ and observe that we can calculate the constant $\tilde{\mathfrak{P}}$ given by (3.39) evaluating the sum of (4.5), (4.6) for $a=b=\lambda_{0}<\min \left(\lambda_{1}, \hat{\lambda}_{1}\right)$. By Figure 4 we conclude that $\tilde{\mathfrak{P}}=0$, where we take $a=b=\lambda_{0}=-4$. Then, according to Theorem 3.8, we can evaluate the difference $\#\left\{\lambda \in \sigma_{2} \mid \lambda \leq b\right\}-\#\left\{\lambda \in \sigma_{1} \mid \lambda \leq a\right\}$ between the numbers of eigenvalues of (4.4) and (4.1) calculating the total number of weighted nodes of the Wronskian for all $i$ and $i^{*}, i=0, \ldots, N, i^{*} \in(i, i+1)$.

Figure 5 presents the graphs of the signs of the Wronskian, $C_{i}(a, b)=q_{i}(a)-\hat{q}_{i}(b)$, $B_{i}(a, b)=\hat{r}_{i}^{-1}(b)-r_{i}^{-1}(a)$ and the relative oscillation numbers $\#\left(Y_{i}^{(0)}(a), \hat{Y}_{i}^{(N+1)}(b)\right)-P_{i}$, $i=0,1,2,3$. For example, we have

$$
\#\left(Y_{0}^{(0)}(a), \hat{Y}_{0}^{(N+1)}(b)\right)-P_{0}=\#_{I I}\left(0, i^{*}\right)-P_{0}+\#_{I}\left(i^{*}, 1\right)=2, \quad i^{*}=1 / 2
$$

because of $B_{0}(a, b)>0, w_{0} w_{i^{*}}<0$, and $C_{0}(a, b)>0, w_{1} w_{i^{*}}<0, i^{*}=1 / 2$. Similarly, in the next point $i=1$, we have $B_{1}(a, b)<0, w_{1} w_{i^{*}}<0$, and $C_{1}(a, b)>0, w_{2} w_{i^{*}}<0, i^{*}=3 / 2$, and then 


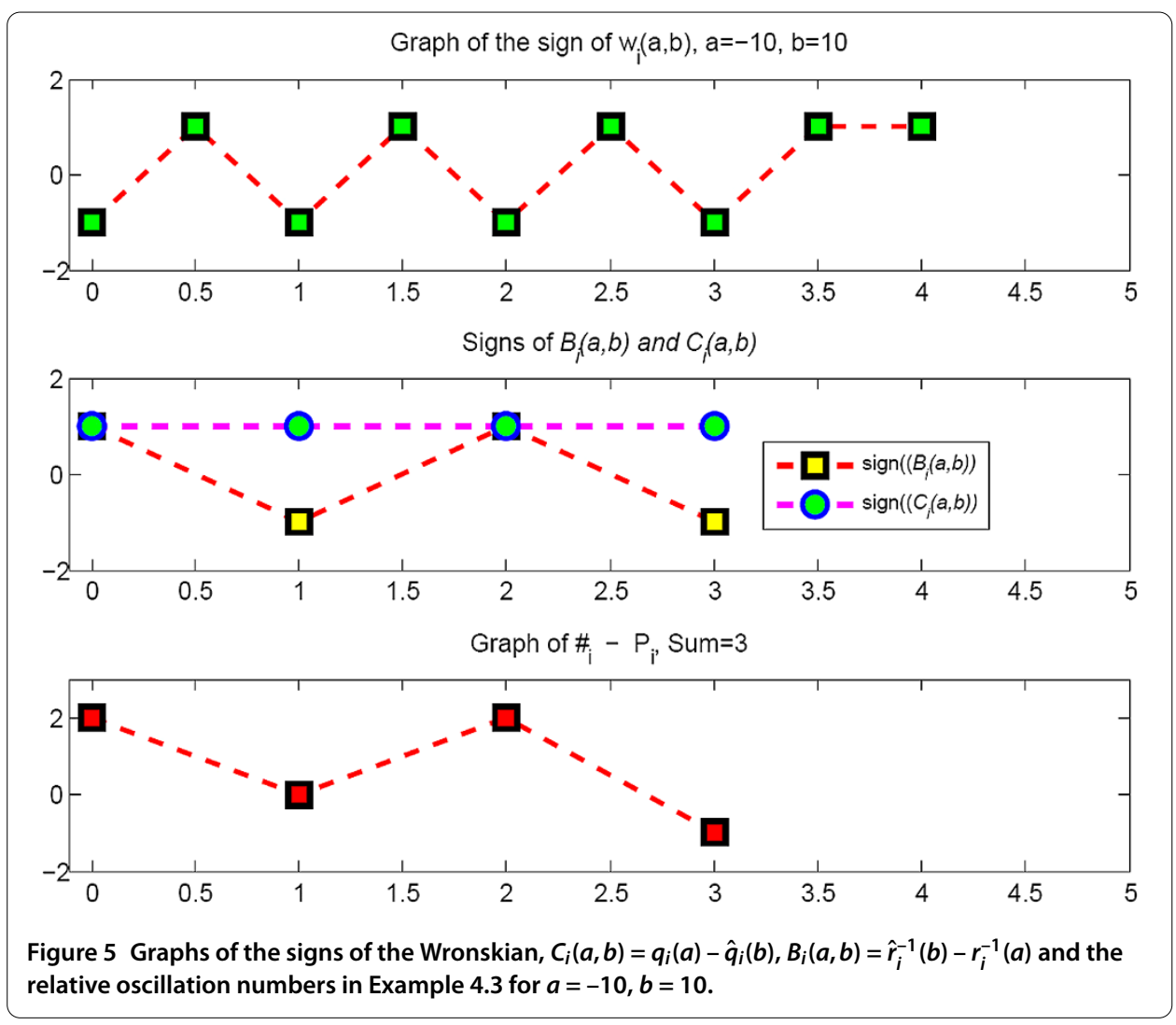

$\#\left(Y_{1}^{(0)}(a), \hat{Y}_{1}^{(N+1)}(b)\right)-P_{1}=-1+1=0$. Finally, according to Figure 3, we have

$$
\#\left\{\lambda \in \sigma_{2} \mid \lambda \leq 10\right\}-\#\left\{\lambda \in \sigma_{1} \mid \lambda \leq-10\right\}=3-0=3,
$$

and the sum of the relative oscillation numbers by Figure 5 equals 3 .

\section{Competing interests}

The author declares that she has no competing interests.

\section{Author's contributions}

The author declares that she has conducted all the research in this paper. She also created the displayed pictures in the software Matlab R210b.

\section{Acknowledgements}

This research was supported by Federal Programme of Ministry of Education and Science of the Russian Federation under Grant 1.6034.2011. The author also thanks anonymous referees for their suggestions which improved the presentation of the results.

Received: 28 June 2013 Accepted: 18 October 2013 Published: 19 Nov 2013

\section{References}

1. Šimon Hilscher, R, Zeidan, V: Symmetric three-term recurrence equations and their symplectic structure. Adv. Differ. Equ. 2010(17), Article ID 626942 (2010)

2. Šimon Hilscher, R: Oscillation theorems for discrete symplectic systems with nonlinear dependence in spectral parameter. Linear Algebra Appl. 437(12), 2922-2960 (2012)

3. Bohner, M, Došlý, O, Kratz, W: An oscillation theorem for discrete eigenvalue problems. Rocky Mt. J. Math. 33(4), 1233-1260 (2003). doi:10.1216/rmjm/1181075460

4. Došlý, O, Kratz, W: Oscillation theorems for symplectic difference systems. J. Differ. Equ. Appl. 13(7), 585-605 (2007). doi:10.1080/10236190701264776

5. Bohner, M, Došlý, O, Kratz, W: Sturmian and spectral theory for discrete symplectic systems. Trans. Am. Math. Soc. 361, 3109-3123 (2009) 
6. Teschl, G: Oscillation theory and renormalized oscillation theory for Jacobi operators. J. Differ. Equ. 129(2), 532-558 (1996). doi:10.1006/jdeq.1996.0126

7. Ammann, K, Teschl, G: Relative oscillation theory for Jacobi matrices. In: Proceedings of the 14th International Conference on Difference Equations and Applications (Istanbul, 2008), pp. 105-115. Uğur-Bahçesşehir University Publishing Company, Istanbul (2009)

8. Krüger, H, Teschl, G: Relative oscillation theory, weighted zeros of the Wronskian, and spectral shift function. Commun. Math. Phys. 287, 613-640 (2009)

9. Krüger, H, Teschl, G: Relative oscillation theory for Sturm-Liouville operators extended. J. Funct. Anal. 254, 1702-1720 (2008)

10. Ammann, K: Relative oscillation theory for Jacobi matrices extended. Oper. Matrices (2013, to appear)

11. Bohner, M, Došlý, O: Disconjugacy and transformations for symplectic systems. Rocky Mt. J. Math. 27, 707-743 (1997)

12. Šimon Hilscher, R: Spectral and oscillation theory for general second order Sturm-Liouville difference equations. Adv. Differ. Equ. 2012, 82 (2012). http://www.advancesindifferenceequations.com/content/2012/1/82

13. Eliseeva, YV: Comparison theorems for symplectic systems of difference equations. Differ. Equ. 46(9), 1339-1352 (2010)

14. Elyseeva, J: On relative oscillation theory for symplectic eigenvalue problems. Appl. Math. Lett. 23, 1231-1237 (2010)

15. Elyseeva, J: A note on relative oscillation theory for symplectic difference systems with general boundary conditions. Appl. Math. Lett. 25, 1809-1814 (2012)

16. Eliseeva, YV: Spectra of discrete symplectic eigenvalue problems with separated boundary conditions. Russ. Math. 55(11), 71-75 (2011)

17. Kratz, W, Šimon Hilscher, R: A generalized index theorem for monotone matrix-valued functions with applications to discrete oscillation theory. SIAM J. Matrix Anal. Appl. 34(1), 228-243 (2013)

18. Elyseeva, J: The comparative index for conjoined bases of symplectic difference systems. In: Difference Equations, Special Functions and Orthogonal Polynomials, Munich, Germany, 25-30 July 2005, pp. 135-145. World Scientific, Singapore (2007)

19. Elyseeva, J: Comparative index for solutions of symplectic difference systems. Differ. Equ. 45, 445-459 (2009)

20. Elyseeva, J: Transformations and the number of focal points for conjoined bases of symplectic difference systems. J. Differ. Equ. Appl. 15, 1055-1066 (2009)

21. Kratz, W: Discrete oscillation. J. Differ. Equ. Appl. 9(1), 135-147 (2003)

22. Elyseeva, J: Symplectic factorizations and the definition of a focal point. In: Proceedings of the Eighth International Conference on Difference Equations and Applications, pp. 127-135. Chapman \& Hall/CRC, Boca Raton (2005)

23. Ahlbrandt, CD, Peterson, AC: Discrete Hamiltonian Systems: Difference Equations, Continued Fractions, and Riccati Equations. Kluwer Academic, Boston (1996)

24. Tian, Y: Equalities and inequalities for inertias of Hermitian matrices with applications. Linear Algebra Appl. 433, 263-296 (2010)

10.1186/1687-1847-2013-328

Cite this article as: Elyseeva: Relative oscillation theory for matrix Sturm-Liouville difference equations extended. Advances in Difference Equations 2013, 2013:328

\section{Submit your manuscript to a SpringerOpen ${ }^{\circ}$ journal and benefit from:}

- Convenient online submission

Rigorous peer review

- Immediate publication on acceptance

Open access: articles freely available online

- High visibility within the field

- Retaining the copyright to your article 